\title{
Positive solutions for a system of Riemann-Liouville fractional boundary value problems with $p$-Laplacian operators
}

Alexandru Tudorache ${ }^{1}$ and Rodica Luca ${ }^{2 *}$ (D)

"Correspondence:

rlucatudor@yahoo.com

${ }^{2}$ Department of Mathematics, Gh.

Asachi Technical University, lasi,

Romania

Full list of author information is

available at the end of the article

\section{Springer}

\begin{abstract}
We study the existence and nonexistence of positive solutions for a system of Riemann-Liouville fractional differential equations with $p$-Laplacian operators, nonnegative nonlinearities and positive parameters, subject to coupled nonlocal boundary conditions which contain Riemann-Stieltjes integrals and various fractional derivatives. We use the Guo-Krasnosel'skii fixed point theorem in the proof of the main existence results.

MSC: 34A08; 34B15; 34B10; 34B18; 45G15

Keywords: Systems of Riemann-Liouville fractional differential equations; Nonlocal coupled boundary conditions; Nonnegative nonlinearities; Existence of solutions; Nonexistence of solutions
\end{abstract}

\section{Introduction}

In this paper we consider the system of nonlinear ordinary fractional differential equations with $\varrho_{1}$-Laplacian and $\varrho_{2}$-Laplacian operators

$$
\begin{cases}D_{0+}^{\alpha_{1}}\left(\varphi_{\varrho_{1}}\left(D_{0+}^{\beta_{1}} x(t)\right)\right)+\lambda f(t, x(t), y(t))=0, & t \in(0,1), \\ D_{0+}^{\alpha_{2}}\left(\varphi_{\varrho_{2}}\left(D_{0+}^{\beta_{2}} y(t)\right)\right)+\mu g(t, x(t), y(t))=0, & t \in(0,1),\end{cases}
$$

with the coupled nonlocal boundary conditions

$$
\left\{\begin{array}{l}
x^{(j)}(0)=0, \quad j=0, \ldots, n-2 ; \quad D_{0+}^{\beta_{1}} x(0)=0, \\
D_{0+}^{\gamma_{0}} x(1)=\sum_{i=1}^{p} \int_{0}^{1} D_{0+}^{\gamma_{i}} y(t) d H_{i}(t), \\
y^{(j)}(0)=0, \quad j=0, \ldots, m-2 ; \quad D_{0+}^{\beta_{2}} y(0)=0, \\
D_{0+}^{\delta_{0}} y(1)=\sum_{i=1}^{q} \int_{0}^{1} D_{0+}^{\delta_{i}} x(t) d K_{i}(t),
\end{array}\right.
$$

where $\alpha_{1}, \alpha_{2} \in(0,1], \beta_{1} \in(n-1, n], \beta_{2} \in(m-1, m], n, m \in \mathbb{N}, n, m \geq 3, p, q \in \mathbb{N}, \gamma_{i} \in \mathbb{R}$ for all $i=0,1, \ldots, p, 0 \leq \gamma_{1}<\gamma_{2}<\cdots<\gamma_{p} \leq \delta_{0}<\beta_{2}-1, \delta_{0} \geq 1, \delta_{i} \in \mathbb{R}$ for all $i=0,1, \ldots, q$, $0 \leq \delta_{1}<\delta_{2}<\cdots<\delta_{q} \leq \gamma_{0}<\beta_{1}-1, \gamma_{0} \geq 1, \varrho_{1}, \varrho_{2}>1, \varphi_{\varrho_{i}}(s)=|s|^{\varrho_{i}-2} s, \varphi_{\varrho_{i}}^{-1}=\varphi_{\rho_{i}}, \frac{1}{\varrho_{i}}+\frac{1}{\rho_{i}}=1$,

(c) The Author(s) 2020. This article is licensed under a Creative Commons Attribution 4.0 International License, which permits use, sharing, adaptation, distribution and reproduction in any medium or format, as long as you give appropriate credit to the original author(s) and the source, provide a link to the Creative Commons licence, and indicate if changes were made. The images or other third party material in this article are included in the article's Creative Commons licence, unless indicated otherwise in a credit line to the material. If material is not included in the article's Creative Commons licence and your intended use is not permitted by statutory regulation or exceeds the permitted use, you will need to obtain permission directly from the copyright holder. To view a copy of this licence, visit http://creativecommons.org/licenses/by/4.0/. 
$i=1,2, \lambda, \mu>0$, the functions $f, g \in C\left([0,1] \times \mathbb{R}_{+} \times \mathbb{R}_{+}, \mathbb{R}_{+}\right)\left(\mathbb{R}_{+}=[0, \infty)\right)$, the integrals from (2) are Riemann-Stieltjes integrals with $H_{i}, i=1, \ldots, p$, and $K_{i}, i=1, \ldots, q$, functions of bounded variation, and $D_{0_{+}}^{k}$ denotes the Riemann-Liouville derivative of order $k$ (for $k=\alpha_{1}, \beta_{1}, \alpha_{2}, \beta_{2}, \gamma_{i}$ for $i=0,1, \ldots, p, \delta_{i}$ for $\left.i=0,1, \ldots, q\right)$.

We present sufficient conditions on the functions $f$ and $g$, and intervals for the parameters $\lambda$ and $\mu$ such that problem (1), (2) has positive solutions. A positive solution of problem (1), (2) is a pair of functions $(x, y) \in\left(C\left([0,1], \mathbb{R}_{+}\right)\right)^{2}$, satisfying (1) and (2) with $x(t)>0$ for all $t \in(0,1]$ or $y(t)>0$ for all $t \in(0,1]$. We also investigate the nonexistence of positive solutions for this problem. The problem (1), (2) is a generalization of the problem studied in [30]. Indeed, if $p=1, q=1, \gamma_{0}=p_{1}, \gamma_{1}=q_{1}, \delta_{0}=p_{2}, \delta_{1}=q_{2}, H_{1}$ is a step function given by $H_{1}(t)=\left\{0, t \in\left[0, \xi_{1}\right) ; a_{1}, t \in\left[\xi_{1}, \xi_{2}\right) ; a_{1}+a_{2}, t \in\left[\xi_{2}, \xi_{3}\right) ; \ldots ; \sum_{i=1}^{N} a_{i}, t \in\right.$ $\left.\left[\xi_{N}, 1\right]\right\}$, and $K_{1}$ is a step function given by $K_{1}(t)=\left\{0, t \in\left[0, \eta_{1}\right) ; b_{1}, t \in\left[\eta_{1}, \eta_{2}\right) ; b_{1}+b_{2}, t \in\right.$ $\left.\left[\eta_{2}, \eta_{3}\right) ; \ldots ; \sum_{i=1}^{M} b_{i}, t \in\left[\eta_{M}, 1\right]\right\}$, then the boundary conditions (2) become the multi-point boundary conditions $(B C)$ from [30]. The system (1) with uncoupled multi-point boundary conditions was studied in [29]. Fractional differential equations and systems of fractional differential equations, subject to various multi-point or Riemann-Stieltjes integral boundary conditions, were studied in the last years in [1-8, 17-25, 28, 31, 37, 39-41]. For various applications of the fractional calculus in several scientific and engineering fields, the reader may consult the books $[10,11,26,27,34-36]$, and the papers $[9,12-$ $15,32,33,38]$.

In Sect. 2, we study a nonlocal boundary value problem for fractional differential equations with $p$-Laplacian operators, and we present some properties of the associated Green functions. Section 3 is devoted to the main existence theorems for the positive solutions with respect to a cone for our problem (1), (2), which are based on the Guo-Krasnosel'skii fixed point theorem (see [16]). In Sect. 4, we present some nonexistence results for the positive solutions of (1), (2), and in Sect. 5, we give an example which illustrates our results.

\section{Preliminary results}

We consider the system of fractional differential equations

$$
\begin{cases}D_{0+}^{\alpha_{1}}\left(\varphi_{\varrho_{1}}\left(D_{0+}^{\beta_{1}} x(t)\right)\right)+\psi(t)=0, & t \in(0,1), \\ D_{0+}^{\alpha_{2}}\left(\varphi_{\varrho_{2}}\left(D_{0+}^{\beta_{2}} y(t)\right)\right)+\chi(t)=0, & t \in(0,1),\end{cases}
$$

with the coupled boundary conditions (2), where $\psi, \chi \in C(0,1) \cap L^{1}(0,1)$.

We denote $\varphi_{\varrho_{1}}\left(D_{0^{+}}^{\beta_{1}} x(t)\right)=u(t)$ and $\varphi_{\varrho_{2}}\left(D_{0^{+}}^{\beta_{2}} y(t)\right)=v(t)$. Then problem (3), (2) is equivalent to the following three problems:

$$
\begin{aligned}
& \left\{\begin{array}{l}
D_{0+}^{\alpha_{1}} u(t)+\psi(t)=0, \quad 0<t<1, \\
u(0)=0,
\end{array}\right. \\
& \left\{\begin{array}{l}
D_{0+}^{\alpha_{2}} v(t)+\chi(t)=0, \quad 0<t<1, \\
v(0)=0,
\end{array}\right.
\end{aligned}
$$


Tudorache and Luce Advances in Difference Equations

(2020) $2020: 292$

Page 3 of 30

and

$$
\begin{cases}D_{0+}^{\beta_{1}} x(t)=\varphi_{\rho_{1}}(u(t)), & t \in(0,1) \\ D_{0+}^{\beta_{2}} y(t)=\varphi_{\rho_{2}}(v(t)), & t \in(0,1)\end{cases}
$$

with the boundary conditions

$$
\left\{\begin{array}{lll}
x^{(j)}(0)=0, & j=0, \ldots, n-2 ; & D_{0+}^{\gamma_{0}} x(1)=\sum_{i=1}^{p} \int_{0}^{1} D_{0+}^{\gamma_{i}} y(t) d H_{i}(t), \\
y^{(j)}(0)=0, & j=0, \ldots, m-2 ; & D_{0+}^{\delta_{0}} y(1)=\sum_{i=1}^{q} \int_{0}^{1} D_{0+}^{\delta_{i}} x(t) d K_{i}(t) .
\end{array}\right.
$$

The first two problems (4) and (5) have the unique solutions $u \in C[0,1]$ and $v \in C[0,1]$, respectively, of the form

$$
u(t)=-I_{0+}^{\alpha_{1}} \psi(t)=-\frac{1}{\Gamma\left(\alpha_{1}\right)} \int_{0}^{t}(t-v)^{\alpha_{1}-1} \psi(v) d v, \quad t \in[0,1],
$$

and

$$
\nu(t)=-I_{0+}^{\alpha_{2}} \chi(t)=-\frac{1}{\Gamma\left(\alpha_{2}\right)} \int_{0}^{t}(t-v)^{\alpha_{2}-1} \chi(v) d v, \quad t \in[0,1],
$$

respectively.

For the third problem (6), (7), we denote

$$
\begin{aligned}
\Delta= & \frac{\Gamma\left(\beta_{1}\right) \Gamma\left(\beta_{2}\right)}{\Gamma\left(\beta_{1}-\gamma_{0}\right) \Gamma\left(\beta_{2}-\delta_{0}\right)}-\left(\sum_{i=1}^{p} \frac{\Gamma\left(\beta_{2}\right)}{\Gamma\left(\beta_{2}-\gamma_{i}\right)} \int_{0}^{1} \tau^{\beta_{2}-\gamma_{i}-1} d H_{i}(\tau)\right) \\
& \times\left(\sum_{i=1}^{q} \frac{\Gamma\left(\beta_{1}\right)}{\Gamma\left(\beta_{1}-\delta_{i}\right)} \int_{0}^{1} \tau^{\beta_{1}-\delta_{i}-1} d K_{i}(\tau)\right) .
\end{aligned}
$$

Lemma 2.1 If $\Delta \neq 0$, then the unique solution $(x, y) \in C[0,1] \times C[0,1]$ of problem (6), (7) is given by

$$
\begin{aligned}
x(t)= & \frac{1}{\Gamma\left(\beta_{1}\right)} \int_{0}^{t}(t-v)^{\beta_{1}-1} \varphi_{\rho_{1}}(u(v)) d v \\
& +\frac{t^{\beta_{1}-1}}{\Delta}\left[-\frac{\Gamma\left(\beta_{2}\right)}{\Gamma\left(\beta_{1}-\gamma_{0}\right) \Gamma\left(\beta_{2}-\delta_{0}\right)} \int_{0}^{1}(1-v)^{\beta_{1}-\gamma_{0}-1} \varphi_{\rho_{1}}(u(v)) d v\right. \\
& +\frac{\Gamma\left(\beta_{2}\right)}{\Gamma\left(\beta_{2}-\delta_{0}\right)} \sum_{i=1}^{p} \frac{1}{\Gamma\left(\beta_{2}-\gamma_{i}\right)} \int_{0}^{1}\left(\int_{0}^{v}(v-\tau)^{\beta_{2}-\gamma_{i}-1} \varphi_{\rho_{2}}(v(\tau)) d \tau\right) d H_{i}(v) \\
& -\left(\sum_{i=1}^{p} \frac{\Gamma\left(\beta_{2}\right)}{\Gamma\left(\beta_{2}-\gamma_{i}\right)} \int_{0}^{1} v^{\beta_{2}-\gamma_{i}-1} d H_{i}(v)\right) \\
& \times\left(\frac{1}{\Gamma\left(\beta_{2}-\delta_{0}\right)} \int_{0}^{1}(1-v)^{\beta_{2}-\delta_{0}-1} \varphi_{\rho_{2}}(v(v)) d v\right) \\
& +\left(\sum_{i=1}^{p} \frac{\Gamma\left(\beta_{2}\right)}{\Gamma\left(\beta_{2}-\gamma_{i}\right)} \int_{0}^{1} v^{\beta_{2}-\gamma_{i}-1} d H_{i}(v)\right)
\end{aligned}
$$




$$
\begin{aligned}
& \left.\times\left(\sum_{i=1}^{q} \frac{1}{\Gamma\left(\beta_{1}-\delta_{i}\right)} \int_{0}^{1}\left(\int_{0}^{v}(v-\tau)^{\beta_{1}-\delta_{i}-1} \varphi_{\rho_{1}}(u(\tau)) d \tau\right) d K_{i}(v)\right)\right] \\
& t \in[0,1] \\
y(t)= & \frac{1}{\Gamma\left(\beta_{2}\right)} \int_{0}^{t}(t-v)^{\beta_{2}-1} \varphi_{\rho_{2}}(v(v)) d v \\
& +\frac{t^{\beta_{2}-1}}{\Delta}\left[-\frac{\Gamma\left(\beta_{1}\right)}{\Gamma\left(\beta_{1}-\gamma_{0}\right) \Gamma\left(\beta_{2}-\delta_{0}\right)} \int_{0}^{1}(1-v)^{\beta_{2}-\delta_{0}-1} \varphi_{\rho_{2}}(v(v)) d v\right. \\
& +\frac{\Gamma\left(\beta_{1}\right)}{\Gamma\left(\beta_{1}-\gamma_{0}\right)} \sum_{i=1}^{q} \frac{1}{\Gamma\left(\beta_{1}-\delta_{i}\right)} \int_{0}^{1}\left(\int_{0}^{v}(v-\tau)^{\beta_{1}-\delta_{i}-1} \varphi_{\rho_{1}}(u(\tau)) d \tau\right) d K_{i}(v) \\
& -\left(\sum_{i=1}^{q} \frac{\Gamma\left(\beta_{1}\right)}{\Gamma\left(\beta_{1}-\delta_{i}\right)} \int_{0}^{1} v^{\beta_{1}-\delta_{i}-1} d K_{i}(v)\right) \\
& \times\left(\frac{1}{\Gamma\left(\beta_{1}-\gamma_{0}\right)} \int_{0}^{1}(1-v)^{\beta_{1}-\gamma_{0}-1} \varphi_{\rho_{1}}(u(v)) d v\right) \\
& +\left(\sum_{i=1}^{q} \frac{\Gamma\left(\beta_{1}\right)}{\Gamma\left(\beta_{1}-\delta_{i}\right)} \int_{0}^{1} v^{\beta_{1}-\delta_{i}-1} d K_{i}(v)\right) \\
& \left.\left(\sum_{i=1}^{p} \frac{1}{\Gamma\left(\beta_{2}-\gamma_{i}\right)} \int_{0}^{1}\left(\int_{0}^{v}(v-\tau)^{\beta_{2}-\gamma_{i}-1} \varphi_{\rho_{2}}(v(\tau)) d \tau\right) d H_{i}(v)\right)\right]
\end{aligned}
$$

$\forall t \in[0,1]$.

Proof The solutions $(x, y) \in\left(C(0,1) \cap L^{1}(0,1)\right)^{2}$ of system $(6)$ are

$$
\begin{aligned}
& x(t)=I_{0+}^{\beta_{1}} \varphi_{\rho_{1}}(u(t))+a_{1} t^{\beta_{1}-1}+a_{2} t^{\beta_{1}-2}+\cdots+a_{n} t^{\beta_{1}-n} \\
& y(t)=I_{0+}^{\beta_{2}} \varphi_{\rho_{2}}(v(t))+b_{1} t^{\beta_{2}-1}+b_{2} t^{\beta_{2}-2}+\cdots+b_{m} t^{\beta_{2}-m}
\end{aligned}
$$

with $a_{1}, a_{2}, \ldots, a_{n}, b_{1}, b_{2}, \ldots, b_{m} \in \mathbb{R}$. By using the boundary conditions $x(0)=x^{\prime}(0)=\cdots=$ $x^{(n-2)}(0)=0$ and $y(0)=y^{\prime}(0)=\cdots=y^{(m-2)}=0$, we obtain $a_{2}=\cdots=a_{n}=0$ and $b_{2}=\cdots=$ $b_{m}=0$. So the above solutions become

$$
\begin{aligned}
& x(t)=I_{0+}^{\beta_{1}} \varphi_{\rho_{1}}(u(t))+a_{1} t^{\beta_{1}-1}=\frac{1}{\Gamma\left(\beta_{1}\right)} \int_{0}^{t}(t-v)^{\beta_{1}-1} \varphi_{\rho_{1}}(u(v)) d v+a_{1} t^{\beta_{1}-1}, \\
& y(t)=I_{0+}^{\beta_{2}} \varphi_{\rho_{2}}(v(t))+b_{1} t^{\beta_{2}-1}=\frac{1}{\Gamma\left(\beta_{2}\right)} \int_{0}^{t}(t-v)^{\beta_{2}-1} \varphi_{\rho_{2}}(v(v)) d v+b_{1} t^{\beta_{2}-1} .
\end{aligned}
$$

For the obtained functions $x$ and $y$ we have

$$
\begin{aligned}
& D_{0+}^{\gamma_{i}} y(t)=b_{1} \frac{\Gamma\left(\beta_{2}\right)}{\Gamma\left(\beta_{2}-\gamma_{i}\right)} t^{\beta_{2}-\gamma_{i}-1}+I_{0+}^{\beta_{1}-\gamma_{i}} \varphi_{\rho_{2}}(v(t)), \quad i=1, \ldots, p, \\
& D_{0+}^{\gamma_{0}} x(t)=a_{1} \frac{\Gamma\left(\beta_{1}\right)}{\Gamma\left(\beta_{1}-\gamma_{0}\right)} t^{\beta_{1}-\gamma_{0}-1}+I_{0+}^{\beta_{1}-\gamma_{0}} \varphi_{\rho_{1}}(u(t)), \\
& D_{0+}^{\delta_{i}} x(t)=a_{1} \frac{\Gamma\left(\beta_{1}\right)}{\Gamma\left(\beta_{1}-\delta_{i}\right)} t^{\beta_{1}-\delta_{i}-1}+I_{0+}^{\beta_{1}-\delta_{i}} \varphi_{\rho_{1}}(u(t)), \quad i=1, \ldots, q, \\
& D_{0+}^{\delta_{0}} y(t)=b_{1} \frac{\Gamma\left(\beta_{2}\right)}{\Gamma\left(\beta_{2}-\delta_{0}\right)} t^{\beta_{2}-\delta_{0}-1}+I_{0+}^{\beta_{2}-\delta_{0}} \varphi_{\rho_{2}}(v(t)),
\end{aligned}
$$




$$
\begin{aligned}
D_{0+}^{\gamma_{0}} x(1) & =a_{1} \frac{\Gamma\left(\beta_{1}\right)}{\Gamma\left(\beta_{1}-\gamma_{0}\right)}+\left.I_{0+}^{\beta_{1}-\gamma_{0}} \varphi_{\rho_{1}}(u(t))\right|_{t=1} \\
& =a_{1} \frac{\Gamma\left(\beta_{1}\right)}{\Gamma\left(\beta_{1}-\gamma_{0}\right)}+\frac{1}{\Gamma\left(\beta_{1}-\gamma_{0}\right)} \int_{0}^{1}(1-v)^{\beta_{1}-\gamma_{0}-1} \varphi_{\rho_{1}}(u(v)) d v, \\
D_{0+}^{\delta_{0}} y(1) & =b_{1} \frac{\Gamma\left(\beta_{2}\right)}{\Gamma\left(\beta_{2}-\delta_{0}\right)}+\left.I_{0+}^{\beta_{2}-\delta_{0}} \varphi_{\rho_{2}}(v(t))\right|_{t=1} \\
& =b_{1} \frac{\Gamma\left(\beta_{2}\right)}{\Gamma\left(\beta_{2}-\delta_{0}\right)}+\frac{1}{\Gamma\left(\beta_{2}-\delta_{0}\right)} \int_{0}^{1}(1-v)^{\beta_{2}-\delta_{0}-1} \varphi_{\rho_{2}}(v(v)) d v .
\end{aligned}
$$

By imposing the boundary conditions $D_{0+}^{\gamma_{0}} x(1)=\sum_{i=1}^{p} \int_{0}^{1} D_{0+}^{\gamma_{i}} y(s) d H_{i}(s)$ and $D_{0+}^{\delta_{0}} y(1)=$ $\sum_{i=1}^{q} \int_{0}^{1} D_{0+}^{\delta_{i}} x(s) d K_{i}(s)$, we deduce the following system in $a_{1}$ and $b_{1}$ :

$$
\left\{\begin{array}{l}
a_{1} \frac{\Gamma\left(\beta_{1}\right)}{\Gamma\left(\beta_{1}-\gamma_{0}\right)}-b_{1} \sum_{i=1}^{p} \int_{0}^{1} \frac{\Gamma\left(\beta_{2}\right)}{\Gamma\left(\beta_{2}-\gamma_{i}\right)} t^{\beta_{2}-\gamma_{i}-1} d H_{i}(t) \\
=-\frac{1}{\Gamma\left(\beta_{1}-\gamma_{0}\right)} \int_{0}^{1}(1-v)^{\beta_{1}-\gamma_{0}-1} \varphi_{\rho_{1}}(u(v)) d v \\
\quad+\sum_{i=1}^{p} \int_{0}^{1} \frac{1}{\Gamma\left(\beta_{2}-\gamma_{i}\right)}\left(\int_{0}^{t}(t-v)^{\beta_{2}-\gamma_{i}-1} \varphi_{\rho_{2}}(v(v)) d v\right) d H_{i}(t) \\
b_{1} \frac{\Gamma\left(\beta_{2}\right)}{\Gamma\left(\beta_{2}-\delta_{0}\right)}-a_{1} \sum_{i=1}^{q} \int_{0}^{1} \frac{\Gamma\left(\beta_{1}\right)}{\Gamma\left(\beta_{1}-\delta_{i}\right)} t^{\beta_{1}-\delta_{i}-1} d K_{i}(t) \\
=-\frac{1}{\Gamma\left(\beta_{2}-\delta_{0}\right)} \int_{0}^{1}(1-v)^{\beta_{2}-\delta_{0}-1} \varphi_{\rho_{2}}(v(v)) d v \\
\quad+\sum_{i=1}^{q} \int_{0}^{1} \frac{1}{\Gamma\left(\beta_{1}-\delta_{i}\right)}\left(\int_{0}^{t}(t-v)^{\beta_{1}-\delta_{i}-1} \varphi_{\rho_{1}}(u(v)) d v\right) d K_{i}(t)
\end{array}\right.
$$

The above system in the unknowns $a_{1}$ and $b_{1}$ has the determinant $\Delta$, which, by the assumption of the lemma, is nonzero. So the system (13) has the unique solution

$$
\begin{aligned}
a_{1}= & -\frac{\Gamma\left(\beta_{2}\right)}{\Delta \Gamma\left(\beta_{1}-\gamma_{0}\right) \Gamma\left(\beta_{2}-\delta_{0}\right)} \int_{0}^{1}(1-v)^{\beta_{1}-\gamma_{0}-1} \varphi_{\rho_{1}}(u(v)) d v \\
& +\frac{\Gamma\left(\beta_{2}\right)}{\Delta \Gamma\left(\beta_{2}-\delta_{0}\right)} \sum_{i=1}^{p} \frac{1}{\Gamma\left(\beta_{2}-\gamma_{i}\right)} \int_{0}^{1}\left(\int_{0}^{t}(t-v)^{\beta_{2}-\gamma_{i}-1} \varphi_{\rho_{2}}(v(v)) d v\right) d H_{i}(t) \\
& -\frac{1}{\Delta}\left(\sum_{i=1}^{p} \frac{\Gamma\left(\beta_{2}\right)}{\Gamma\left(\beta_{2}-\gamma_{i}\right)} \int_{0}^{1} t^{\beta_{2}-\gamma_{i}-1} d H_{i}(t)\right) \\
& \times\left(\frac{1}{\Gamma\left(\beta_{2}-\delta_{0}\right)} \int_{0}^{1}(1-v)^{\beta_{2}-\delta_{0}-1} \varphi_{\rho_{2}}(v(v)) d v\right) \\
& +\frac{1}{\Delta}\left(\sum_{i=1}^{p} \frac{\Gamma\left(\beta_{2}\right)}{\Gamma\left(\beta_{2}-\gamma_{i}\right)} \int_{0}^{1} t^{\beta_{2}-\gamma_{i}-1} d H_{i}(t)\right) \\
& \times\left(\sum_{i=1}^{q} \frac{1}{\Gamma\left(\beta_{1}-\delta_{i}\right)} \int_{0}^{1}\left(\int_{0}^{t}(t-v)^{\beta_{1}-\delta_{i}-1} \varphi_{\rho_{1}}(u(v)) d v\right) d K_{i}(t)\right) \\
b_{1}= & -\frac{\Gamma\left(\beta_{1}\right)}{\Delta \Gamma\left(\beta_{1}-\gamma_{0}\right) \Gamma\left(\beta_{2}-\delta_{0}\right)} \int_{0}^{1}(1-v)^{\beta_{2}-\delta_{0}-1} \varphi_{\rho_{2}}(v(v)) d v \\
& +\frac{\Gamma\left(\beta_{1}\right)}{\Delta \Gamma\left(\beta_{1}-\gamma_{0}\right)} \sum_{i=1}^{q} \frac{1}{\Gamma\left(\beta_{1}-\delta_{i}\right)} \int_{0}^{1}\left(\int_{0}^{t}(t-v)^{\beta_{1}-\delta_{i}-1} \varphi_{\rho_{1}}(u(v)) d v\right) d K_{i}(t) \\
& -\frac{1}{\Delta}\left(\sum_{i=1}^{q} \frac{\Gamma\left(\beta_{1}\right)}{\Gamma\left(\beta_{1}-\delta_{i}\right)} \int_{0}^{1} t^{\beta_{1}-\delta_{i}-1} d K_{i}(t)\right)
\end{aligned}
$$


Tudorache and Luck Advances in Difference Equations

(2020) 2020:292

Page 6 of 30

$$
\begin{aligned}
& \times\left(\frac{1}{\Gamma\left(\beta_{1}-\gamma_{0}\right)} \int_{0}^{1}(1-v)^{\beta_{1}-\gamma_{0}-1} \varphi_{\rho_{1}}(u(v)) d v\right) \\
& +\frac{1}{\Delta}\left(\sum_{i=1}^{q} \frac{\Gamma\left(\beta_{1}\right)}{\Gamma\left(\beta_{1}-\delta_{i}\right)} \int_{0}^{1} t^{\beta_{1}-\delta_{i}-1} d K_{i}(t)\right) \\
& \times\left(\sum_{i=1}^{p} \frac{1}{\Gamma\left(\beta_{2}-\gamma_{i}\right)} \int_{0}^{1}\left(\int_{0}^{t}(t-v)^{\beta_{2}-\gamma_{i}-1} \varphi_{\rho_{2}}(v(v)) d v\right) d H_{i}(t)\right) .
\end{aligned}
$$

Now replacing the constants $a_{1}$ and $b_{1}$ given by (14) in (12) we find the solution $(x, y) \in$ $C[0,1] \times C[0,1]$ of problem (6), (7) presented in (11). Conversely, we can easily prove that the functions $x, y$ given by (11) satisfy the problem (6), (7).

Lemma 2.2 If $\Delta \neq 0$, then the solution ( $x, y)$ of problem (6), (7) given by (11) can be written as

$$
\begin{cases}x(t)=-\int_{0}^{1} \mathcal{G}_{1}(t, v) \varphi_{\rho_{1}}(u(v)) d v-\int_{0}^{1} \mathcal{G}_{2}(t, v) \varphi_{\rho_{2}}(v(v)) d v, & \forall t \in[0,1] \\ y(t)=-\int_{0}^{1} \mathcal{G}_{3}(t, v) \varphi_{\rho_{1}}(u(v)) d v-\int_{0}^{1} \mathcal{G}_{4}(t, v) \varphi_{\rho_{2}}(v(v)) d v, & \forall t \in[0,1]\end{cases}
$$

where

$$
\begin{aligned}
\mathcal{G}_{1}(t, v)= & g_{1}(t, v)+\frac{t^{\beta_{1}-1}}{\Delta}\left(\sum_{i=1}^{p} \frac{\Gamma\left(\beta_{2}\right)}{\Gamma\left(\beta_{2}-\gamma_{i}\right)} \int_{0}^{1} s^{\beta_{2}-\gamma_{i}-1} d H_{i}(s)\right) \\
& \times\left(\sum_{i=1}^{q} \int_{0}^{1} g_{1 i}(\tau, v) d K_{i}(\tau)\right), \\
\mathcal{G}_{2}(t, v)= & \frac{t^{\beta_{1}-1} \Gamma\left(\beta_{2}\right)}{\Delta \Gamma\left(\beta_{2}-\delta_{0}\right)} \sum_{i=1}^{p} \int_{0}^{1} g_{2 i}(\tau, v) d H_{i}(\tau), \\
\mathcal{G}_{3}(t, v)= & \frac{t^{\beta_{2}-1} \Gamma\left(\beta_{1}\right)}{\Delta \Gamma\left(\beta_{1}-\gamma_{0}\right)} \sum_{i=1}^{q} \int_{0}^{1} g_{1 i}(\tau, v) d K_{i}(\tau), \\
\mathcal{G}_{4}(t, v)= & g_{2}(t, v)+\frac{t^{\beta_{2}-1}}{\Delta}\left(\sum_{i=1}^{q} \frac{\Gamma\left(\beta_{1}\right)}{\Gamma\left(\beta_{1}-\delta_{i}\right)} \int_{0}^{1} s^{\beta_{1}-\delta_{i}-1} d K_{i}(s)\right) \\
& \times\left(\sum_{i=1}^{p} \int_{0}^{1} g_{2 i}(\tau, v) d H_{i}(\tau)\right),
\end{aligned}
$$

for all $(t, v) \in[0,1] \times[0,1]$ and

$$
\begin{aligned}
& g_{1}(t, s)=\frac{1}{\Gamma\left(\beta_{1}\right)} \begin{cases}t^{\beta_{1}-1}(1-s)^{\beta_{1}-\gamma_{0}-1}-(t-s)^{\beta_{1}-1}, & 0 \leq s \leq t \leq 1, \\
t^{\beta_{1}-1}(1-s)^{\beta_{1}-\gamma_{0}-1}, & 0 \leq t \leq s \leq 1,\end{cases} \\
& g_{1 i}(\tau, s)=\frac{1}{\Gamma\left(\beta_{1}-\delta_{i}\right)} \begin{cases}\tau^{\beta_{1}-\delta_{i}-1}(1-s)^{\beta_{1}-\gamma_{0}-1}-(\tau-s)^{\beta_{1}-\delta_{i}-1}, & 0 \leq s \leq \tau \leq 1, \\
\tau^{\beta_{1}-\delta_{i}-1}(1-s)^{\beta_{1}-\gamma_{0}-1}, & 0 \leq \tau \leq s \leq 1,\end{cases} \\
& g_{2}(t, s)=\frac{1}{\Gamma\left(\beta_{2}\right)} \begin{cases}t^{\beta_{2}-1}(1-s)^{\beta_{2}-\delta_{0}-1}-(t-s)^{\beta_{2}-1}, & 0 \leq s \leq t \leq 1, \\
t^{\beta_{2}-1}(1-s)^{\beta_{2}-\delta_{0}-1}, & 0 \leq t \leq s \leq 1,\end{cases}
\end{aligned}
$$


Tudorache and Luck Advances in Difference Equations

(2020) 2020:292

Page 7 of 30

$$
g_{2 j}(\tau, s)=\frac{1}{\Gamma\left(\beta_{2}-\gamma_{j}\right)} \begin{cases}\tau^{\beta_{2}-\gamma_{j}-1}(1-s)^{\beta_{2}-\delta_{0}-1}-(\tau-s)^{\beta_{2}-\gamma_{j}-1}, & 0 \leq s \leq \tau \leq 1, \\ \tau^{\beta_{2}-\gamma_{j}-1}(1-s)^{\beta_{2}-\delta_{0}-1}, & 0 \leq \tau \leq s \leq 1\end{cases}
$$

for all $i=1, \ldots, q$ and $j=1, \ldots, p$.

Proof For $x(t)$ from (11) we have

$$
\begin{aligned}
& x(t)=\frac{1}{\Gamma\left(\beta_{1}\right)} \int_{0}^{t}(t-s)^{\beta_{1}-1} \varphi_{\rho_{1}}(u(s)) d s \\
& -\frac{t^{\beta_{1}-1} \Gamma\left(\beta_{2}\right)}{\Delta \Gamma\left(\beta_{1}-\gamma_{0}\right) \Gamma\left(\beta_{2}-\delta_{0}\right)} \int_{0}^{1}(1-s)^{\beta_{1}-\gamma_{0}-1} \varphi_{\rho_{1}}(u(s)) d s \\
& +\frac{t^{\beta_{1}}-1}{\Delta}\left(\sum_{i=1}^{p} \frac{\Gamma\left(\beta_{2}\right)}{\Gamma\left(\beta_{2}-\gamma_{i}\right)} \int_{0}^{1} s^{\beta_{2}-\gamma_{i}-1} d H_{i}(s)\right) \\
& \times\left(\sum_{i=1}^{q} \frac{1}{\Gamma\left(\beta_{1}-\delta_{i}\right)} \int_{0}^{1}\left(\int_{0}^{s}(s-\tau)^{\beta_{1}-\delta_{i}-1} \varphi_{\rho_{1}}(u(\tau)) d \tau\right) d K_{i}(s)\right) \\
& +\frac{t^{\beta_{1}-1} \Gamma\left(\beta_{2}\right)}{\Delta \Gamma\left(\beta_{2}-\delta_{0}\right)} \sum_{i=1}^{p} \frac{1}{\Gamma\left(\beta_{2}-\gamma_{i}\right)} \int_{0}^{1}\left(\int_{0}^{s}(s-\tau)^{\beta_{2}-\gamma_{i}-1} \varphi_{\rho_{2}}(v(\tau)) d \tau\right) d H_{i}(s) \\
& -\frac{t^{\beta_{1}-1}}{\Delta}\left(\sum_{i=1}^{p} \frac{\Gamma\left(\beta_{2}\right)}{\Gamma\left(\beta_{2}-\gamma_{i}\right)} \int_{0}^{1} s^{\beta_{2}-\gamma_{i}-1} d H_{i}(s)\right) \\
& \times\left(\frac{1}{\Gamma\left(\beta_{2}-\delta_{0}\right)} \int_{0}^{1}(1-s)^{\beta_{2}-\delta_{0}-1} \varphi_{\rho_{2}}(v(s)) d s\right) \\
& =-\frac{1}{\Gamma\left(\beta_{1}\right)} \int_{0}^{t}\left[t^{\beta_{1}-1}(1-s)^{\beta_{1}-\gamma_{0}-1}-(t-s)^{\beta_{1}-1}\right] \varphi_{\rho_{1}}(u(s)) d s \\
& -\frac{1}{\Gamma\left(\beta_{1}\right)} \int_{t}^{1} t^{\beta_{1}-1}(1-s)^{\beta_{1}-\gamma_{0}-1} \varphi_{\rho_{1}}(u(s)) d s \\
& +\frac{1}{\Gamma\left(\beta_{1}\right)} \int_{0}^{1} t^{\beta_{1}-1}(1-s)^{\beta_{1}-\gamma_{0}-1} \varphi_{\rho_{1}}(u(s)) d s \\
& -\frac{t^{\beta_{1}-1} \Gamma\left(\beta_{2}\right)}{\Delta \Gamma\left(\beta_{1}-\gamma_{0}\right) \Gamma\left(\beta_{2}-\delta_{0}\right)} \int_{0}^{1}(1-s)^{\beta_{1}-\gamma_{0}-1} \varphi_{\rho_{1}}(u(s)) d s \\
& +\frac{t^{\beta_{1}-1}}{\Delta}\left(\sum_{i=1}^{p} \frac{\Gamma\left(\beta_{2}\right)}{\Gamma\left(\beta_{2}-\gamma_{i}\right)} \int_{0}^{1} s^{\beta_{2}-\gamma_{i}-1} d H_{i}(s)\right) \\
& \times\left(\sum_{i=1}^{q} \frac{1}{\Gamma\left(\beta_{1}-\delta_{i}\right)} \int_{0}^{1}\left(\int_{0}^{s}(s-\tau)^{\beta_{1}-\delta_{i}-1} \varphi_{\rho_{1}}(u(\tau)) d \tau\right) d K_{i}(s)\right) \\
& +\frac{t^{\beta_{1}-1} \Gamma\left(\beta_{2}\right)}{\Delta \Gamma\left(\beta_{2}-\delta_{0}\right)} \sum_{i=1}^{p} \frac{1}{\Gamma\left(\beta_{2}-\gamma_{i}\right)} \int_{0}^{1}\left(\int_{\tau}^{1}(s-\tau)^{\beta_{2}-\gamma_{i}-1} d H_{i}(s)\right) \varphi_{\rho_{2}}(v(\tau)) d \tau \\
& -\frac{t^{\beta_{1}-1}}{\Delta \Gamma\left(\beta_{2}-\delta_{0}\right)} \sum_{i=1}^{p} \frac{\Gamma\left(\beta_{2}\right)}{\Gamma\left(\beta_{2}-\gamma_{i}\right)} \int_{0}^{1}\left(\int_{0}^{1} \tau^{\beta_{2}-\gamma_{i}-1} d H_{i}(\tau)\right) \\
& \times(1-s)^{\beta_{2}-\delta_{0}-1} \varphi_{\rho_{2}}(v(s)) d s \\
& =-\int_{0}^{1} g_{1}(t, s) \varphi_{\rho_{1}}(u(s)) d s+\frac{1}{\Gamma\left(\beta_{1}\right)} \int_{0}^{1} t^{\beta_{1}-1}(1-s)^{\beta_{1}-\gamma_{0}-1} \varphi_{\rho_{1}}(u(s)) d s
\end{aligned}
$$


Tudorache and Luck Advances in Difference Equations

(2020) 2020:292

Page 8 of 30

$$
\begin{aligned}
& -\frac{t^{\beta_{1}-1} \Gamma\left(\beta_{2}\right)}{\Delta \Gamma\left(\beta_{1}-\gamma_{0}\right) \Gamma\left(\beta_{2}-\delta_{0}\right)} \int_{0}^{1}(1-s)^{\beta_{1}-\gamma_{0}-1} \varphi_{\rho_{1}}(u(s)) d s \\
& +\frac{t^{\beta_{1}-1}}{\Delta}\left(\sum_{i=1}^{p} \frac{\Gamma\left(\beta_{2}\right)}{\Gamma\left(\beta_{2}-\gamma_{i}\right)} \int_{0}^{1} s^{\beta_{2}-\gamma_{i}-1} d H_{i}(s)\right) \\
& \times\left(\sum_{i=1}^{q} \frac{1}{\Gamma\left(\beta_{1}-\delta_{i}\right)} \int_{0}^{1}\left(\int_{0}^{s}(s-\tau)^{\beta_{1}-\delta_{i}-1} \varphi_{\rho_{1}}(u(\tau)) d \tau\right) d K_{i}(s)\right) \\
& -\frac{t^{\beta_{1}-1} \Gamma\left(\beta_{2}\right)}{\Delta \Gamma\left(\beta_{2}-\delta_{0}\right)} \sum_{i=1}^{p} \frac{1}{\Gamma\left(\beta_{2}-\gamma_{i}\right)}\left[-\int_{0}^{1}\left(\int_{s}^{1}(\tau-s)^{\beta_{2}-\gamma_{i}-1} d H_{i}(\tau)\right) \varphi_{\rho_{2}}(v(s)) d s\right. \\
& \left.+\int_{0}^{1}\left(\int_{0}^{1} \tau^{\beta_{2}-\gamma_{i}-1}(1-s)^{\beta_{2}-\delta_{0}-1} d H_{i}(\tau)\right) \varphi_{\rho_{2}}(v(s)) d s\right], \quad \forall t \in[0,1] .
\end{aligned}
$$

Then we deduce

$$
\begin{aligned}
& x(t)=-\int_{0}^{1} g_{1}(t, s) \varphi_{\rho_{1}}(u(s)) d s \\
& -\frac{1}{\Delta}\left(\sum_{i=1}^{p} \frac{\Gamma\left(\beta_{2}\right)}{\Gamma\left(\beta_{2}-\gamma_{i}\right)} \int_{0}^{1} s^{\beta_{2}-\gamma_{i}-1} d H_{i}(s)\right)\left(\sum_{i=1}^{q} \frac{\Gamma\left(\beta_{1}\right)}{\Gamma\left(\beta_{1}-\delta_{i}\right)} \int_{0}^{1} s^{\beta_{1}-\delta_{i}-1} d K_{i}(s)\right) \\
& \times \frac{1}{\Gamma\left(\beta_{1}\right)} \int_{0}^{1} t^{\beta_{1}-1}(1-s)^{\beta_{1}-\gamma_{0}-1} \varphi_{\rho_{1}}(u(s)) d s \\
& +\frac{t^{\beta_{1}-1}}{\Delta}\left(\sum_{i=1}^{p} \frac{\Gamma\left(\beta_{2}\right)}{\Gamma\left(\beta_{2}-\gamma_{i}\right)} \int_{0}^{1} s^{\beta_{2}-\gamma_{i}-1} d H_{i}(s)\right) \\
& \times\left(\sum_{i=1}^{q} \frac{1}{\Gamma\left(\beta_{1}-\delta_{i}\right)} \int_{0}^{1}\left(\int_{0}^{s}(s-\tau)^{\beta_{1}-\delta_{i}-1} \varphi_{\rho_{1}}(u(\tau)) d \tau\right) d K_{i}(s)\right) \\
& -\frac{t^{\beta_{1}-1} \Gamma\left(\beta_{2}\right)}{\Delta \Gamma\left(\beta_{2}-\delta_{0}\right)} \sum_{i=1}^{p} \int_{0}^{1}\left(\int_{0}^{1} g_{2 i}(\tau, s) d H_{i}(\tau)\right) \varphi_{\rho_{2}}(v(s)) d s \\
& =-\int_{0}^{1} g_{1}(t, s) \varphi_{\rho_{1}}(u(s)) d s-\frac{t^{\beta_{1}-1}}{\Delta}\left(\sum_{i=1}^{p} \frac{\Gamma\left(\beta_{2}\right)}{\Gamma\left(\beta_{2}-\gamma_{i}\right)} \int_{0}^{1} s^{\beta_{2}-\gamma_{i}-1} d H_{i}(s)\right) \\
& \times\left[\sum_{i=1}^{q} \frac{1}{\Gamma\left(\beta_{1}-\delta_{i}\right)}\left(\int_{0}^{1} \tau^{\beta_{1}-\delta_{i}-1} d K_{i}(\tau)\right)\left(\int_{0}^{1}(1-s)^{\beta_{1}-\gamma_{0}-1} \varphi_{\rho_{1}}(u(s)) d s\right)\right. \\
& \left.-\sum_{i=1}^{q} \frac{1}{\Gamma\left(\beta_{1}-\delta_{i}\right)} \int_{0}^{1}\left(\int_{0}^{s}(s-\tau)^{\beta_{1}-\delta_{i}-1} \varphi_{\rho_{1}}(u(\tau)) d \tau\right) d K_{i}(s)\right] \\
& -\int_{0}^{1}\left(\frac{t^{\beta_{1}-1} \Gamma\left(\beta_{2}\right)}{\Delta \Gamma\left(\beta_{2}-\delta_{0}\right)} \sum_{i=1}^{p} \int_{0}^{1} g_{2 i}(\tau, s) d H_{i}(\tau)\right) \varphi_{\rho_{2}}(v(s)) d s \\
& =-\int_{0}^{1} g_{1}(t, s) \varphi_{\rho_{1}}(u(s)) d s-\frac{t^{\beta_{1}-1}}{\Delta}\left(\sum_{i=1}^{p} \frac{\Gamma\left(\beta_{2}\right)}{\Gamma\left(\beta_{2}-\gamma_{i}\right)} \int_{0}^{1} s^{\beta_{2}-\gamma_{i}-1} d H_{i}(s)\right) \\
& \times\left\{\sum _ { i = 1 } ^ { q } \frac { 1 } { \Gamma ( \beta _ { 1 } - \delta _ { i } ) } \left[\int_{0}^{1}\left(\int_{0}^{1} \tau^{\beta_{1}-\delta_{i}-1}(1-s)^{\beta_{1}-\gamma_{0}-1} d K_{i}(\tau)\right) \varphi_{\rho_{1}}(u(s)) d s\right.\right. \\
& \left.\left.-\int_{0}^{1}\left(\int_{s}^{1}(\tau-s)^{\beta_{1}-\delta_{i}-1} d K_{i}(\tau)\right) \varphi_{\rho_{1}}(u(s)) d s\right]\right\}
\end{aligned}
$$


Tudorache and Luck Advances in Difference Equations

(2020) 2020:292

Page 9 of 30

$$
\begin{aligned}
& -\int_{0}^{1}\left(\frac{t^{\beta_{1}-1} \Gamma\left(\beta_{2}\right)}{\Delta \Gamma\left(\beta_{2}-\delta_{0}\right)} \sum_{i=1}^{p} \int_{0}^{1} g_{2 i}(\tau, s) d H_{i}(\tau)\right) \varphi_{\rho_{2}}(v(s)) d s \\
= & -\int_{0}^{1} g_{1}(t, s) \varphi_{\rho_{1}}(u(s)) d s-\frac{t^{\beta_{1}-1}}{\Delta}\left(\sum_{i=1}^{p} \frac{\Gamma\left(\beta_{2}\right)}{\Gamma\left(\beta_{2}-\gamma_{i}\right)} \int_{0}^{1} s^{\beta_{2}-\gamma_{i}-1} d H_{i}(s)\right) \\
& \times\left[\sum_{i=1}^{q} \int_{0}^{1}\left(\int_{0}^{1} g_{1 i}(\tau, s) d K_{i}(\tau)\right) \varphi_{\rho_{1}}(u(s)) d s\right] \\
& -\int_{0}^{1}\left(\frac{t^{\beta_{1}-1} \Gamma\left(\beta_{2}\right)}{\Delta \Gamma\left(\beta_{2}-\delta_{0}\right)} \sum_{i=1}^{p} \int_{0}^{1} g_{2 i}(\tau, s) d H_{i}(\tau)\right) \varphi_{\rho_{2}}(v(s)) d s \\
= & -\int_{0}^{1} \mathcal{G}_{1}(t, s) \varphi_{\rho_{1}}(u(s)) d s-\int_{0}^{1} \mathcal{G}_{2}(t, s) \varphi_{\rho_{2}}(v(s)) d s, \quad \forall t \in[0,1],
\end{aligned}
$$

where $g_{1}, g_{1 i}, i=1, \ldots, q, g_{2 i}, i=1, \ldots, p$, are given by (17), and $\mathcal{G}_{1}$ and $\mathcal{G}_{2}$ are given by (16).

For $y(t)$ we find

$$
\begin{aligned}
& y(t)=\frac{t^{\beta_{2}-1} \Gamma\left(\beta_{1}\right)}{\Delta \Gamma\left(\beta_{1}-\gamma_{0}\right)} \sum_{i=1}^{q} \frac{1}{\Gamma\left(\beta_{1}-\delta_{i}\right)} \int_{0}^{1}\left(\int_{0}^{s}(s-\tau)^{\beta_{1}-\delta_{i}-1} \varphi_{\rho_{1}}(u(\tau)) d \tau\right) d K_{i}(s) \\
& -\frac{t^{\beta_{2}-1}}{\Delta}\left(\sum_{i=1}^{q} \frac{\Gamma\left(\beta_{1}\right)}{\Gamma\left(\beta_{1}-\delta_{i}\right)} \int_{0}^{1} s^{\beta_{1}-\delta_{i}-1} d K_{i}(s)\right) \\
& \times\left(\frac{1}{\Gamma\left(\beta_{1}-\gamma_{0}\right)} \int_{0}^{1}(1-s)^{\beta_{1}-\gamma_{0}-1} \varphi_{\rho_{1}}(u(s)) d s\right) \\
& +\frac{1}{\Gamma\left(\beta_{2}\right)} \int_{0}^{t}(t-s)^{\beta_{2}-1} \varphi_{\rho_{2}}(v(s)) d s \\
& -\frac{t^{\beta_{2}-1} \Gamma\left(\beta_{1}\right)}{\Delta \Gamma\left(\beta_{1}-\gamma_{0}\right) \Gamma\left(\beta_{2}-\delta_{0}\right)} \int_{0}^{1}(1-s)^{\beta_{2}-\delta_{0}-1} \varphi_{\rho_{2}}(v(s)) d s \\
& +\frac{t^{\beta_{2}-1}}{\Delta}\left(\sum_{i=1}^{q} \frac{\Gamma\left(\beta_{1}\right)}{\Gamma\left(\beta_{1}-\delta_{i}\right)} \int_{0}^{1} s^{\beta_{1}-\delta_{i}-1} d K_{i}(s)\right) \\
& \times\left(\sum_{i=1}^{p} \frac{1}{\Gamma\left(\beta_{2}-\gamma_{i}\right)} \int_{0}^{1}\left(\int_{0}^{s}(s-\tau)^{\beta_{2}-\gamma_{i}-1} \varphi_{\rho_{2}}(v(\tau)) d \tau\right) d H_{i}(s)\right) \\
& =\frac{t^{\beta_{2}-1} \Gamma\left(\beta_{1}\right)}{\Delta \Gamma\left(\beta_{1}-\gamma_{0}\right)} \sum_{i=1}^{q} \frac{1}{\Gamma\left(\beta_{1}-\delta_{i}\right)} \int_{0}^{1}\left(\int_{\tau}^{1}(s-\tau)^{\beta_{1}-\delta_{i}-1} d K_{i}(s)\right) \varphi_{\rho_{1}}(u(\tau)) d \tau \\
& -\frac{t^{\beta_{2}-1}}{\Delta} \sum_{i=1}^{q} \frac{\Gamma\left(\beta_{1}\right)}{\Gamma\left(\beta_{1}-\delta_{i}\right) \Gamma\left(\beta_{1}-\gamma_{0}\right)} \\
& \times \int_{0}^{1}\left(\int_{0}^{1} \tau^{\beta_{1}-\delta_{i}-1}(1-s)^{\beta_{1}-\gamma_{0}-1} d K_{i}(\tau)\right) \varphi_{\rho_{1}}(u(s)) d s \\
& -\frac{1}{\Gamma\left(\beta_{2}\right)} \int_{0}^{t}\left[t^{\beta_{2}-1}(1-s)^{\beta_{2}-\delta_{0}-1}-(t-s)^{\beta_{2}-1}\right] \varphi_{\rho_{2}}(v(s)) d s \\
& -\frac{1}{\Gamma\left(\beta_{2}\right)} \int_{t}^{1} t^{\beta_{2}-1}(1-s)^{\beta_{2}-\delta_{0}-1} \varphi_{\rho_{2}}(v(s)) d s \\
& +\frac{1}{\Gamma\left(\beta_{2}\right)} \int_{0}^{1} t^{\beta_{2}-1}(1-s)^{\beta_{2}-\delta_{0}-1} \varphi_{\rho_{2}}(v(s)) d s
\end{aligned}
$$


Tudorache and Luck Advances in Difference Equations

(2020) 2020:292

Page 10 of 30

$$
\begin{aligned}
& -\frac{t^{\beta_{2}-1} \Gamma\left(\beta_{1}\right)}{\Delta \Gamma\left(\beta_{1}-\gamma_{0}\right) \Gamma\left(\beta_{2}-\delta_{0}\right)} \int_{0}^{1}(1-s)^{\beta_{2}-\delta_{0}-1} \varphi_{\rho_{2}}(v(s)) d s \\
& +\frac{t^{\beta_{2}-1}}{\Delta}\left(\sum_{i=1}^{q} \frac{\Gamma\left(\beta_{1}\right)}{\Gamma\left(\beta_{1}-\delta_{i}\right)} \int_{0}^{1} s^{\beta_{1}-\delta_{i}-1} d K_{i}(s)\right) \\
& \times\left(\sum_{i=1}^{p} \frac{1}{\Gamma\left(\beta_{2}-\gamma_{i}\right)} \int_{0}^{1}\left(\int_{0}^{s}(s-\tau)^{\beta_{2}-\gamma_{i}-1} \varphi_{\rho_{2}}(v(\tau)) d \tau\right) d H_{i}(s)\right) \\
& =\frac{t^{\beta_{2}-1} \Gamma\left(\beta_{1}\right)}{\Delta \Gamma\left(\beta_{1}-\gamma_{0}\right)} \sum_{i=1}^{q} \frac{1}{\Gamma\left(\beta_{1}-\delta_{i}\right)} \int_{0}^{1}\left(\int_{s}^{1}(\tau-s)^{\beta_{1}-\delta_{i}-1} d K_{i}(\tau)\right) \varphi_{\rho_{1}}(u(s)) d s \\
& -\frac{t^{\beta_{2}-1} \Gamma\left(\beta_{1}\right)}{\Delta \Gamma\left(\beta_{1}-\gamma_{0}\right)} \sum_{i=1}^{q} \frac{1}{\Gamma\left(\beta_{1}-\delta_{i}\right)} \\
& \times \int_{0}^{1}\left(\int_{0}^{1} \tau^{\beta_{1}-\delta_{i}-1}(1-s)^{\beta_{1}-\gamma_{0}-1} d K_{i}(\tau)\right) \varphi_{\rho_{1}}(u(s)) d s \\
& -\int_{0}^{1} g_{2}(t, s) \varphi_{\rho_{2}}(v(s)) d s+\frac{1}{\Gamma\left(\beta_{2}\right)} \int_{0}^{1} t^{\beta_{2}-1}(1-s)^{\beta_{2}-\delta_{0}-1} \varphi_{\rho_{2}}(v(s)) d s \\
& -\frac{t^{\beta_{2}-1} \Gamma\left(\beta_{1}\right)}{\Delta \Gamma\left(\beta_{1}-\gamma_{0}\right) \Gamma\left(\beta_{2}-\delta_{0}\right)} \int_{0}^{1}(1-s)^{\beta_{2}-\delta_{0}-1} \varphi_{\rho_{2}}(v(s)) d s \\
& +\frac{t^{\beta_{2}-1}}{\Delta}\left(\sum_{i=1}^{q} \frac{\Gamma\left(\beta_{1}\right)}{\Gamma\left(\beta_{1}-\delta_{i}\right)} \int_{0}^{1} s^{\beta_{1}-\delta_{i}-1} d K_{i}(s)\right) \\
& \times\left(\sum_{i=1}^{p} \frac{1}{\Gamma\left(\beta_{2}-\gamma_{i}\right)} \int_{0}^{1}\left(\int_{0}^{s}(s-\tau)^{\beta_{2}-\gamma_{i}-1} \varphi_{\rho_{2}}(v(\tau)) d \tau\right) d H_{i}(s)\right), \quad \forall t \in[0,1] .
\end{aligned}
$$

Hence we obtain

$$
\begin{aligned}
y(t)= & -\frac{t^{\beta_{2}-1} \Gamma\left(\beta_{1}\right)}{\Delta \Gamma\left(\beta_{1}-\gamma_{0}\right)} \sum_{i=1}^{q} \int_{0}^{1}\left(\int_{0}^{1} g_{1 i}(\tau, s) d K_{i}(\tau)\right) \varphi_{\rho_{1}}(u(s)) d s \\
& -\int_{0}^{1} g_{2}(t, s) \varphi_{\rho_{2}}(v(s)) d s \\
& -\frac{1}{\Delta}\left(\sum_{i=1}^{p} \frac{\Gamma\left(\beta_{2}\right)}{\Gamma\left(\beta_{2}-\gamma_{i}\right)} \int_{0}^{1} s^{\beta_{2}-\gamma_{i}-1} d H_{i}(s)\right)\left(\sum_{i=1}^{q} \frac{\Gamma\left(\beta_{1}\right)}{\Gamma\left(\beta_{1}-\delta_{i}\right)} \int_{0}^{1} s^{\beta_{1}-\delta_{i}-1} d K_{i}(s)\right) \\
& \times \frac{1}{\Gamma\left(\beta_{2}\right)} \int_{0}^{1} t^{\beta_{2}-1}(1-s)^{\beta_{2}-\delta_{0}-1} \varphi_{\rho_{2}}(v(s)) d s \\
& +\frac{t^{\beta_{2}-1}\left(\sum_{i=1}^{q} \frac{\Gamma\left(\beta_{1}\right)}{\Gamma\left(\beta_{1}-\delta_{i}\right)} \int_{0}^{1} s^{\beta_{1}-\delta_{i}-1} d K_{i}(s)\right)}{\Delta} \\
& \times\left(\sum_{i=1}^{p} \frac{1}{\Gamma\left(\beta_{2}-\gamma_{i}\right)} \int_{0}^{1}\left(\int_{0}^{s}(s-\tau)^{\beta_{2}-\gamma_{i}-1} \varphi_{\rho_{2}}(v(\tau)) d \tau\right) d H_{i}(s)\right) \\
= & \frac{t^{\beta_{2}-1} \Gamma\left(\beta_{1}\right)}{\Delta \Gamma\left(\beta_{1}-\gamma_{0}\right)} \sum_{i=1}^{q} \int_{0}^{1}\left(\int_{0}^{1} g_{1 i}(\tau, s) d K_{i}(\tau)\right) \varphi_{\rho_{1}}(u(s)) d s \\
& -\int_{0}^{1} g_{2}(t, s) \varphi_{\rho_{2}}(v(s)) d s-\frac{t^{\beta_{2}-1}}{\Delta}\left(\sum_{i=1}^{q} \frac{\Gamma\left(\beta_{1}\right)}{\Gamma\left(\beta_{1}-\delta_{i}\right)} \int_{0}^{1} s^{\beta_{1}-\delta_{i}-1} d K_{i}(s)\right)
\end{aligned}
$$




$$
\begin{aligned}
& \times\left[\sum_{i=1}^{p} \frac{1}{\Gamma\left(\beta_{2}-\gamma_{i}\right)} \int_{0}^{1}\left(\int_{0}^{1} \tau^{\beta_{2}-\gamma_{i}-1}(1-s)^{\beta_{2}-\delta_{0}-1} d H_{i}(\tau)\right) \varphi_{\rho_{2}}(v(s)) d s\right. \\
& \left.-\sum_{i=1}^{p} \frac{1}{\Gamma\left(\beta_{2}-\gamma_{i}\right)} \int_{0}^{1}\left(\int_{\tau}^{1}(s-\tau)^{\beta_{2}-\gamma_{i}-1} d H_{i}(s)\right) \varphi_{\rho_{2}}(v(\tau)) d \tau\right] \\
= & -\frac{t^{\beta_{2}-1} \Gamma\left(\beta_{1}\right)}{\Delta \Gamma\left(\beta_{1}-\gamma_{0}\right)} \sum_{i=1}^{q} \int_{0}^{1}\left(\int_{0}^{1} g_{1 i}(\tau, s) d K_{i}(\tau)\right) \varphi_{\rho_{1}}(u(s)) d s \\
& -\int_{0}^{1} g_{2}(t, s) \varphi_{\rho_{2}}(v(s)) d s-\frac{t^{\beta_{2}-1}}{\Delta}\left(\sum_{i=1}^{q} \frac{\Gamma\left(\beta_{1}\right)}{\Gamma\left(\beta_{1}-\delta_{i}\right)} \int_{0}^{1} s^{\beta_{1}-\delta_{i}-1} d K_{i}(s)\right) \\
& \times\left\{\sum _ { i = 1 } ^ { p } \frac { 1 } { \Gamma ( \beta _ { 2 } - \gamma _ { i } ) } \left[\int_{0}^{1}\left(\int_{0}^{1} \tau^{\beta_{2}-\gamma_{i}-1}(1-s)^{\beta_{2}-\delta_{0}-1} d H_{i}(\tau)\right) \varphi_{\rho_{2}}(v(s)) d s\right.\right. \\
& \left.\left.-\int_{0}^{1}\left(\int_{s}^{1}(\tau-s)^{\beta_{2}-\gamma_{i}-1} d H_{i}(\tau)\right) \varphi_{\rho_{2}}(v(s)) d s\right]\right\} \\
= & -\frac{t^{\beta_{2}-1} \Gamma\left(\beta_{1}\right)}{\Delta \Gamma\left(\beta_{1}-\gamma_{0}\right)} \sum_{i=1}^{q} \int_{0}^{1}\left(\int_{0}^{1} g_{1 i}(\tau, s) d K_{i}(\tau)\right) \varphi_{\rho_{1}}(u(s)) d s \\
& -\int_{0}^{1} g_{2}(t, s) \varphi_{\rho_{2}}(v(s)) d s-\frac{t^{\beta_{2}-1}}{\Delta}\left(\sum_{i=1}^{q} \frac{\Gamma\left(\beta_{1}\right)}{\Gamma\left(\beta_{1}-\delta_{i}\right)} \int_{0}^{1} s^{\beta_{1}-\delta_{i}-1} d K_{i}(s)\right) \\
= & -\int_{0}^{1} \mathcal{G}_{3}(t, s) \varphi_{\rho_{1}}(u(s)) d s-\int_{0}^{p} \mathcal{G}_{4}(t, s) \varphi_{\rho_{2}}(v(s)) d s, \quad \forall t \in[0,1], \\
& {\left.\left[\int_{0}^{1} g_{2 i}(\tau, s) d H_{i}(\tau)\right) \varphi_{\rho_{2}}(v(s)) d s\right] }
\end{aligned}
$$

where $g_{1 i}, i=1, \ldots, q, g_{2}, g_{2 i}, i=1, \ldots, p$, are given by (17), and $\mathcal{G}_{3}$ and $\mathcal{G}_{4}$ are given by (16).

Therefore, by (8), (9) and (15) we deduce the following lemma.

Lemma 2.3 If $\Delta \neq 0$, then the unique solution $(x, y) \in C[0,1] \times C[0,1]$ of problem (3), (2) is given by

$$
\begin{cases}x(t)=\int_{0}^{1} \mathcal{G}_{1}(t, v) \varphi_{\rho_{1}}\left(I_{0+}^{\alpha_{1}} \psi(v)\right) d v+\int_{0}^{1} \mathcal{G}_{2}(t, v) \varphi_{\rho_{2}}\left(I_{0+}^{\alpha_{2}} \chi(v)\right) d v, & \forall t \in[0,1] \\ y(t)=\int_{0}^{1} \mathcal{G}_{3}(t, v) \varphi_{\rho_{1}}\left(I_{0+}^{\alpha_{1}} \psi(v)\right) d v+\int_{0}^{1} \mathcal{G}_{4}(t, v) \varphi_{\rho_{2}}\left(I_{0+}^{\alpha_{2}} \chi(v)\right) d v, & \forall t \in[0,1]\end{cases}
$$

In the next lemma, we present some properties of the functions $g_{1}, g_{2}, g_{1 i}, i=1, \ldots, q$, and $g_{2 i}, i=1, \ldots, p$.

Lemma 2.4 ([19]) The functions $g_{1}, g_{2}, g_{1 i}, i=1, \ldots, q, g_{2 i}, i=1, \ldots, p$, given by (17) have the properties:

(a) The functions $g_{1}, g_{2}, g_{1 i}, i=1, \ldots, q, g_{2 i}, i=1, \ldots, p$, are continuous on $[0,1] \times[0,1]$; $g_{1}(t, v) \geq 0, g_{2}(t, v) \geq 0, g_{1 i}(t, v) \geq 0, i=1, \ldots, q, g_{2 i}(t, v) \geq 0, i=1, \ldots, p$, for all $(t, v) \in[0,1] \times[0,1] ; g_{1}(t, v)>0, g_{2}(t, v)>0, g_{1 i}(t, v)>0, i=1, \ldots, q, g_{2 i}(t, v)>0$, $i=1, \ldots, p$, for all $(t, v) \in(0,1) \times(0,1)$;

(b) $g_{1}(t, v) \leq h_{1}(v)$ for all $(t, v) \in[0,1] \times[0,1]$, where $h_{1}(v)=\frac{1}{\Gamma\left(\beta_{1}\right)}(1-v)^{\beta_{1}-\gamma_{0}-1}\left(1-(1-v)^{\gamma_{0}}\right), \forall v \in[0,1]$; 
(c) $g_{1}(t, v) \geq t^{\beta_{1}-1} h_{1}(v)$ for all $(t, v) \in[0,1] \times[0,1]$;

(d) $g_{2}(t, v) \leq h_{2}(v)$ for all $(t, v) \in[0,1] \times[0,1]$, where $h_{2}(v)=\frac{1}{\Gamma\left(\beta_{2}\right)}(1-v)^{\beta_{2}-\delta_{0}-1}\left(1-(1-v)^{\delta_{0}}\right), \forall v \in[0,1]$;

(e) $g_{2}(t, v) \geq t^{\beta_{2}-1} h_{2}(v)$ for all $(t, v) \in[0,1] \times[0,1]$;

(f) $g_{1 i}(t, v) \leq \frac{1}{\Gamma\left(\beta_{1}-\delta_{i}\right)} t^{\beta_{1}-\delta_{i}-1}(1-v)^{\beta_{1}-\gamma_{0}-1}$ for all $(t, v) \in[0,1] \times[0,1], i=1, \ldots, q$;

(g) $g_{1 i}(t, v) \geq t^{\beta_{1}-\delta_{i}-1} h_{1 i}(v)$ for all $(t, v) \in[0,1] \times[0,1]$, where $h_{1 i}(v)=\frac{1}{\Gamma\left(\beta_{1}-\delta_{i}\right)}(1-v)^{\beta_{1}-\gamma_{0}-1}\left(1-(1-v)^{\gamma_{0}-\delta_{i}}\right), \forall v \in[0,1], i=1, \ldots, q ;$

(h) $g_{2 i}(t, v) \leq \frac{1}{\Gamma\left(\beta_{2}-\gamma_{i}\right)} t^{\beta_{2}-\gamma_{i}-1}(1-v)^{\beta_{2}-\delta_{0}-1}$ for all $(t, v) \in[0,1] \times[0,1], i=1, \ldots, p$;

(i) $g_{2 i}(t, v) \geq t^{\beta_{2}-\gamma_{i}-1} h_{2 i}(v)$ for all $(t, v) \in[0,1] \times[0,1]$, where $h_{2 i}(v)=\frac{1}{\Gamma\left(\beta_{2}-\gamma_{i}\right)}(1-v)^{\beta_{2}-\delta_{0}-1}\left(1-(1-v)^{\delta_{0}-\gamma_{i}}\right), \forall v \in[0,1], i=1, \ldots, p$.

By using Lemma 2.4, we obtain the following properties for the functions $\mathcal{G}_{i}, i=1, \ldots, 4$.

Lemma 2.5 Assume that $\Delta>0, H_{i}, i=1, \ldots, p, K_{i}, i=1, \ldots, q$, are nondecreasing functions. Then the functions $\mathcal{G}_{i}, i=1, \ldots, 4$, given by $(16)$ have the properties:

(a) $\mathcal{G}_{i}:[0,1] \times[0,1] \rightarrow[0, \infty), i=1, \ldots, 4$, are continuous functions;

(b) $\mathcal{G}_{1}(t, v) \leq \mathcal{J}_{1}(v)$ for all $(t, v) \in[0,1] \times[0,1]$, where

$$
\begin{aligned}
\mathcal{J}_{1}(v)= & h_{1}(v)+\frac{1}{\Delta}\left(\sum_{i=1}^{p} \frac{\Gamma\left(\beta_{2}\right)}{\Gamma\left(\beta_{2}-\gamma_{i}\right)} \int_{0}^{1} s^{\beta_{2}-\gamma_{i}-1} d H_{i}(s)\right) \\
& \times\left(\sum_{i=1}^{q} \int_{0}^{1} g_{1 i}(\tau, v) d K_{i}(\tau)\right), \quad \forall v \in[0,1] ;
\end{aligned}
$$

(c) $\mathcal{G}_{1}(t, v) \geq t^{\beta_{1}-1} \mathcal{J}_{1}(v)$ for all $(t, v) \in[0,1] \times[0,1]$;

(d) $\mathcal{G}_{2}(t, v) \leq \mathcal{J}_{2}(v)$, for all $(t, v) \in[0,1] \times[0,1]$, where

$$
\mathcal{J}_{2}(v)=\frac{\Gamma\left(\beta_{2}\right)}{\Delta \Gamma\left(\beta_{2}-\delta_{0}\right)} \sum_{i=1}^{p} \int_{0}^{1} g_{2 i}(\tau, v) d H_{i}(\tau), \quad \forall v \in[0,1] ;
$$

(e) $\mathcal{G}_{2}(t, v)=t^{\beta_{1}-1} \mathcal{J}_{2}(v)$ for all $(t, v) \in[0,1] \times[0,1]$;

(f) $\mathcal{G}_{3}(t, v) \leq \mathcal{J}_{3}(v)$ for all $(t, v) \in[0,1] \times[0,1]$, where

$$
\mathcal{J}_{3}(v)=\frac{\Gamma\left(\beta_{1}\right)}{\Delta \Gamma\left(\beta_{1}-\gamma_{0}\right)} \sum_{i=1}^{q} \int_{0}^{1} g_{1 i}(\tau, v) d K_{i}(\tau), \quad \forall v \in[0,1] ;
$$

(g) $\mathcal{G}_{3}(t, v)=t^{\beta_{2}-1} \mathcal{J}_{3}(v)$ for all $(t, v) \in[0,1] \times[0,1]$;

(h) $\mathcal{G}_{4}(t, v) \leq \mathcal{J}_{4}(v)$ for all $(t, v) \in[0,1] \times[0,1]$, where

$$
\begin{aligned}
\mathcal{J}_{4}(v)= & h_{2}(v)+\frac{1}{\Delta}\left(\sum_{i=1}^{q} \frac{\Gamma\left(\beta_{1}\right)}{\Gamma\left(\beta_{1}-\delta_{i}\right)} \int_{0}^{1} s^{\beta_{1}-\delta_{i}-1} d K_{i}(s)\right) \\
& \times\left(\sum_{i=1}^{p} \int_{0}^{1} g_{2 i}(\tau, v) d H_{i}(\tau)\right), \quad \forall v \in[0,1] ;
\end{aligned}
$$

(i) $\mathcal{G}_{4}(t, v) \geq t^{\beta_{2}-1} \mathcal{J}_{4}(v)$, for all $(t, v) \in[0,1] \times[0,1]$. 


\section{Existence of positive solutions}

In this section we present sufficient conditions for the functions $f$ and $g$, and intervals for the parameters $\lambda$ and $\mu$ such that problem (1), (2) has at least one positive solution.

We give now the basic assumptions that we will use in the main results.

(I1) $\alpha_{1}, \alpha_{2} \in(0,1], \beta_{1} \in(n-1, n], \beta_{2} \in(m-1, m], n, m \in \mathbb{N}, n, m \geq 3, p, q \in \mathbb{N}, \gamma_{i} \in \mathbb{R}$ for all $i=0,1, \ldots, p, 0 \leq \gamma_{1}<\gamma_{2}<\cdots<\gamma_{p} \leq \delta_{0}<\beta_{2}-1, \delta_{0} \geq 1, \delta_{i} \in \mathbb{R}$ for all $i=$ $0,1, \ldots, q, 0 \leq \delta_{1}<\delta_{2}<\cdots<\delta_{q} \leq \gamma_{0}<\beta_{1}-1, \gamma_{0} \geq 1, H_{i}:[0,1] \rightarrow \mathbb{R}, i=1, \ldots, p$, and $K_{j}:[0,1] \rightarrow \mathbb{R}, j=1, \ldots, q$, are nondecreasing functions, $\exists i_{0} \in\{1, \ldots, p\}$, such that $H_{i_{0}}(1)>H_{i_{0}}(0), \exists j_{0} \in\{1, \ldots, q\}$, such that $K_{j_{0}}(1)>K_{j_{0}}(0), \lambda>0, \mu>0, \Delta>0(\Delta$ is given by (10)), $\varrho_{i}>1, \varphi_{\varrho_{i}}(s)=|s|^{\varrho_{i}-2} s, \varphi_{\varrho_{i}}^{-1}=\varphi_{\rho_{i}}, \rho_{i}=\frac{\varrho_{i}}{\varrho_{i}-1}, i=1,2$.

(I2) The functions $f, g:[0,1] \times \mathbb{R}_{+} \times \mathbb{R}_{+} \rightarrow \mathbb{R}_{+}$are continuous.

For $\left[\theta_{1}, \theta_{2}\right] \subset[0,1]$ with $0<\theta_{1}<\theta_{2} \leq 1$, we introduce the following extreme limits:

$$
\begin{aligned}
& \mathfrak{f}_{0}^{s}=\lim _{\substack{x+y \rightarrow 0^{+} \\
x, y \geq 0}} \max _{\substack{t \in[0,1] \\
\varphi_{\varrho_{1}}(x+y)}} \frac{f(t, x, y)}{\varphi^{s}}=\limsup _{\substack{x+y \rightarrow 0^{+} \\
x, y \geq 0}} \max _{t \in[0,1]} \frac{g(t, x, y)}{\varphi_{\varrho_{2}}(x+y)},
\end{aligned}
$$

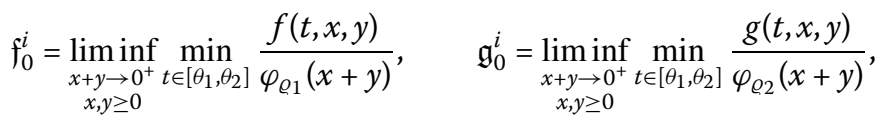

$$
\begin{aligned}
& \mathfrak{f}_{\infty}^{s}=\limsup _{\substack{x+y \rightarrow \infty \\
x, y \geq 0}} \max _{\substack{t \in[0,1] \\
\varphi_{\varrho_{1}}(x+y)}} \frac{f(t, x, y)}{\mathfrak{g}_{\infty}^{s}}=\limsup _{\substack{x+y \rightarrow \infty \\
x, y \geq 0}} \max _{t \in[0,1]} \frac{g(t, x, y)}{\varphi_{\varrho_{2}}(x+y)},
\end{aligned}
$$

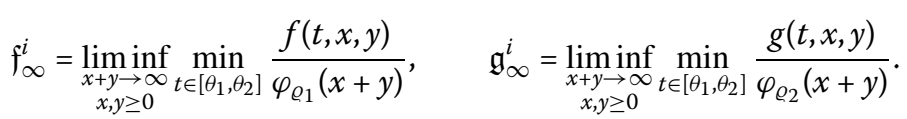

By using Lemma 2.3 (Eqs. (18)), $(x, y)$ is a solution of problem (1), (2) if and only if $(x, y)$ is a solution of the following nonlinear system of integral equations:

$$
\left\{\begin{aligned}
x(t)= & \lambda^{\rho_{1}-1} \int_{0}^{1} \mathcal{G}_{1}(t, v) \varphi_{\rho_{1}}\left(I_{0+}^{\alpha_{1}} f(v, x(v), y(v))\right) d v \\
& +\mu^{\rho_{2}-1} \int_{0}^{1} \mathcal{G}_{2}(t, v) \varphi_{\rho_{2}}\left(I_{0+}^{\alpha_{2}} g(v, x(v), y(v))\right) d v, \quad t \in[0,1], \\
y(t)= & \lambda^{\rho_{1}-1} \int_{0}^{1} \mathcal{G}_{3}(t, v) \varphi_{\rho_{1}}\left(I_{0+}^{\alpha_{1}} f(v, x(v), y(v))\right) d v \\
& +\mu^{\rho_{2}-1} \int_{0}^{1} \mathcal{G}_{4}(t, v) \varphi_{\rho_{2}}\left(I_{0+}^{\alpha_{2}} g(v, x(v), y(v))\right) d v, \quad t \in[0,1] .
\end{aligned}\right.
$$

We consider the Banach space $\mathcal{X}=C[0,1]$ with the supremum norm

$$
\|x\|=\sup _{t \in[0,1]}|x(t)|
$$

and the Banach space $\mathcal{Y}=\mathcal{X} \times \mathcal{X}$ with the norm $\|(x, y)\| \mathcal{Y}=\|x\|+\|y\|$. We define the cones

$$
\begin{aligned}
& \mathcal{P}_{1}=\left\{x \in \mathcal{X}, x(t) \geq t^{\beta_{1}-1}\|x\|, \forall t \in[0,1]\right\} \subset \mathcal{X}, \\
& \mathcal{P}_{2}=\left\{y \in \mathcal{X}, y(t) \geq t^{\beta_{2}-1}\|y\|, \forall t \in[0,1]\right\} \subset \mathcal{X},
\end{aligned}
$$

and $\mathcal{P}=\mathcal{P}_{1} \times \mathcal{P}_{2} \subset \mathcal{Y}$. 
We also define the operators $\mathcal{A}_{1}, \mathcal{A}_{2}: \mathcal{Y} \rightarrow \mathcal{X}$ and $\mathcal{A}: \mathcal{Y} \rightarrow \mathcal{Y}$ by

$$
\begin{aligned}
\mathcal{A}_{1}(x, y)(t)= & \lambda^{\rho_{1}-1} \int_{0}^{1} \mathcal{G}_{1}(t, v) \varphi_{\rho_{1}}\left(I_{0+}^{\alpha_{1}} f(v, x(v), y(v))\right) d v \\
& +\mu^{\rho_{2}-1} \int_{0}^{1} \mathcal{G}_{2}(t, v) \varphi_{\rho_{2}}\left(I_{0+}^{\alpha_{2}} g(v, x(v), y(v))\right) d v, \quad t \in[0,1], \\
\mathcal{A}_{2}(x, y)(t)= & \lambda^{\rho_{1}-1} \int_{0}^{1} \mathcal{G}_{3}(t, v) \varphi_{\rho_{1}}\left(I_{0+}^{\alpha_{1}} f(v, x(v), y(v))\right) d v \\
& +\mu^{\rho_{2}-1} \int_{0}^{1} \mathcal{G}_{4}(t, v) \varphi_{\rho_{2}}\left(I_{0+}^{\alpha_{2}} g(v, x(v), y(v))\right) d v, \quad t \in[0,1],
\end{aligned}
$$

and $\mathcal{A}(x, y)=\left(\mathcal{A}_{1}(x, y), \mathcal{A}_{2}(x, y)\right),(x, y) \in \mathcal{Y}$. The pair $(x, y)$ is a solution of problem (1), (2) if and only if $(x, y)$ is a fixed point of the operator $\mathcal{A}$.

Lemma 3.1 If (I1) and (I2) hold, then $\mathcal{A}: \mathcal{P} \rightarrow \mathcal{P}$ is a completely continuous operator.

Proof Let $(x, y) \in \mathcal{P}$ be an arbitrary element. Because $\mathcal{A}_{1}(x, y)$ and $\mathcal{A}_{2}(x, y)$ satisfy the problem (3), (2) for $\psi(t)=\lambda f(t, x(t), y(t))$ and $\chi(t)=\mu g(t, x(t), y(t)), t \in[0,1]$, then by Lemma 2.5 we obtain

$$
\begin{aligned}
\mathcal{A}_{1}(x, y)(t) \leq & \lambda^{\rho_{1}-1} \int_{0}^{1} \mathcal{J}_{1}(v) \varphi_{\rho_{1}}\left(I_{0+}^{\alpha_{1}} f(v, x(v), y(v))\right) d v \\
& +\mu^{\rho_{2}-1} \int_{0}^{1} \mathcal{J}_{2}(v) \varphi_{\rho_{2}}\left(I_{0+}^{\alpha_{2}} g(v, x(v), y(v))\right) d v, \quad \forall t \in[0,1], \\
\mathcal{A}_{2}(x, y)(t) \leq & \lambda^{\rho_{1}-1} \int_{0}^{1} \mathcal{J}_{3}(v) \varphi_{\rho_{1}}\left(I_{0+}^{\alpha_{1}} f(v, x(v), y(v))\right) d v \\
& +\mu^{\rho_{2}-1} \int_{0}^{1} \mathcal{J}_{4}(v) \varphi_{\rho_{2}}\left(I_{0+}^{\alpha_{2}} g(v, x(v), y(v))\right) d v, \quad \forall t \in[0,1],
\end{aligned}
$$

and then

$$
\begin{aligned}
\left\|\mathcal{A}_{1}(x, y)\right\| \leq & \lambda^{\rho_{1}-1} \int_{0}^{1} \mathcal{J}_{1}(v) \varphi_{\rho_{1}}\left(I_{0+}^{\alpha_{1}} f(v, x(v), y(v))\right) d v \\
& +\mu^{\rho_{2}-1} \int_{0}^{1} \mathcal{J}_{2}(v) \varphi_{\rho_{2}}\left(I_{0+}^{\alpha_{2}} g(v, x(v), y(v))\right) d v \\
\left\|\mathcal{A}_{2}(x, y)\right\| \leq & \lambda^{\rho_{1}-1} \int_{0}^{1} \mathcal{J}_{3}(v) \varphi_{\rho_{1}}\left(I_{0+}^{\alpha_{1}} f(v, x(v), y(v))\right) d v \\
& +\mu^{\rho_{2}-1} \int_{0}^{1} \mathcal{J}_{4}(v) \varphi_{\rho_{2}}\left(I_{0+}^{\alpha_{2}} g(v, x(v), y(v))\right) d v .
\end{aligned}
$$

Hence by using again Lemma 2.5, we deduce

$$
\begin{aligned}
\mathcal{A}_{1}(x, y)(t) \geq & \lambda^{\rho_{1}-1} \int_{0}^{1} t^{\beta_{1}-1} \mathcal{J}_{1}(v) \varphi_{\rho_{1}}\left(I_{0+}^{\alpha_{1}} f(v, x(v), y(v))\right) d v \\
& +\mu^{\rho_{2}-1} \int_{0}^{1} t^{\beta_{1}-1} \mathcal{J}_{2}(v) \varphi_{\rho_{2}}\left(I_{0+}^{\alpha_{2}} g(v, x(v), y(v))\right) d v \\
\geq & t^{\beta_{1}-1}\left\|\mathcal{A}_{1}(x, y)\right\|, \quad \forall t \in[0,1],
\end{aligned}
$$




$$
\begin{aligned}
\mathcal{A}_{2}(x, y)(t) \geq & \lambda^{\varrho_{1}-1} \int_{0}^{1} t^{\beta_{2}-1} \mathcal{J}_{3}(v) \varphi_{\rho_{1}}\left(I_{0_{+}}^{\alpha_{1}} f(v, x(v), y(v))\right) d v \\
& +\mu^{\rho_{2}-1} \int_{0}^{1} t^{\beta_{2}-1} \mathcal{J}_{4}(v) \varphi_{\rho_{2}}\left(I_{0+}^{\alpha_{2}} g(v, x(v), y(v))\right) d v \\
\geq & t^{\beta_{2}-1}\left\|\mathcal{A}_{2}(x, y)\right\|, \quad \forall t \in[0,1] .
\end{aligned}
$$

Therefore $\mathcal{A}(x, y)=\left(\mathcal{A}_{1}(x, y), \mathcal{A}_{2}(x, y)\right) \in \mathcal{P}$, and then $\mathcal{A}(\mathcal{P}) \subset \mathcal{P}$. By using the continuity of the functions $f, g, \mathcal{G}_{i}, i=1, \ldots, 4$, and the Ascoli-Arzela theorem, we can prove that $\mathcal{A}_{1}$ and $\mathcal{A}_{2}$ are completely continuous operators (compact operators, that is, they map bounded sets into relatively compact sets, and continuous), and then $\mathcal{A}$ is a completely continuous operator.

For $\left[\theta_{1}, \theta_{2}\right] \subset[0,1]$ with $0<\theta_{1}<\theta_{2} \leq 1$, we denote

$$
\begin{aligned}
& L_{1}=\frac{1}{\left(\Gamma\left(\alpha_{1}+1\right)\right)^{\rho_{1}-1}} \int_{0}^{1} v^{\alpha_{1}\left(\rho_{1}-1\right)} \mathcal{J}_{1}(v) d v \\
& L_{2}=\frac{1}{\left(\Gamma\left(\alpha_{2}+1\right)\right)^{\rho_{2}-1}} \int_{0}^{1} v^{\alpha_{2}\left(\rho_{2}-1\right)} \mathcal{J}_{2}(v) d v \\
& L_{3}=\frac{1}{\left(\Gamma\left(\alpha_{1}+1\right)\right)^{\rho_{1}-1}} \int_{0}^{1} v^{\alpha_{1}\left(\rho_{1}-1\right)} \mathcal{J}_{3}(v) d v \\
& L_{4}=\frac{1}{\left(\Gamma\left(\alpha_{2}+1\right)\right)^{\rho_{2}-1}} \int_{0}^{1} v^{\alpha_{2}\left(\rho_{2}-1\right)} \mathcal{J}_{4}(v) d v \\
& \widetilde{L}_{1}=\frac{1}{\left(\Gamma\left(\alpha_{1}+1\right)\right)^{\rho_{1}-1}} \int_{\theta_{1}}^{\theta_{2}}\left(v-\theta_{1}\right)^{\alpha_{1}\left(\rho_{1}-1\right)} \mathcal{J}_{1}(v) d v, \\
& \widetilde{L}_{2}=\frac{1}{\left(\Gamma\left(\alpha_{2}+1\right)\right)^{\rho_{2}-1}} \int_{\theta_{1}}^{\theta_{2}}\left(v-\theta_{1}\right)^{\alpha_{2}\left(\rho_{2}-1\right)} \mathcal{J}_{2}(v) d v \\
& \widetilde{L}_{3}=\frac{1}{\left(\Gamma\left(\alpha_{1}+1\right)\right)^{\rho_{1}-1}} \int_{\theta_{1}}^{\theta_{2}}\left(v-\theta_{1}\right)^{\alpha_{1}\left(\rho_{1}-1\right)} \mathcal{J}_{3}(v) d v, \\
& \widetilde{L}_{4}=\frac{1}{\left(\Gamma\left(\alpha_{2}+1\right)\right)^{\rho_{2}-1}} \int_{\theta_{1}}^{\theta_{2}}\left(v-\theta_{1}\right)^{\alpha_{2}\left(\rho_{2}-1\right)} \mathcal{J}_{4}(v) d v,
\end{aligned}
$$

where $\mathcal{J}_{i}, i=1, \ldots, 4$, are defined in Lemma 2.5 .

For $\mathfrak{f}_{0}^{s}, \mathfrak{g}_{0}^{s}, \mathfrak{f}_{\infty}^{i}, \mathfrak{g}_{\infty}^{i} \in(0, \infty)$ and numbers $c_{1}, c_{2} \in[0,1], c_{3}, c_{4} \in(0,1), a \in[0,1]$ and $b \in$ $(0,1)$, we introduce the numbers

$$
\begin{aligned}
& A=\max \left\{\frac{1}{\mathfrak{f}_{\infty}^{i}} \varphi_{\varrho_{1}}\left(\frac{a c_{1}}{\zeta \zeta_{1} \widetilde{L}_{1}}\right), \frac{1}{\mathfrak{f}_{\infty}^{i}} \varphi_{\varrho_{1}}\left(\frac{(1-a) c_{2}}{\zeta \zeta_{2} \widetilde{L}_{3}}\right)\right\}, \\
& B=\min \left\{\frac{1}{\mathfrak{f}_{0}^{s}} \varphi_{\varrho_{1}}\left(\frac{b c_{3}}{L_{1}}\right), \frac{1}{\mathfrak{f}_{0}^{s}} \varphi_{\varrho_{1}}\left(\frac{(1-b) c_{4}}{L_{3}}\right)\right\}, \\
& C=\max \left\{\frac{1}{\mathfrak{g}_{\infty}^{i}} \varphi_{\varrho_{2}}\left(\frac{a\left(1-c_{1}\right)}{\zeta \zeta_{1}}\right), \frac{1}{\mathfrak{g}_{\infty}^{i}} \varphi_{\varrho_{2}}\left(\frac{(1-a)\left(1-c_{2}\right)}{\zeta \zeta_{2} \widetilde{L}_{4}}\right)\right\}, \\
& D=\min \left\{\frac{1}{\mathfrak{g}_{0}^{s}} \varphi_{\varrho_{2}}\left(\frac{b\left(1-c_{3}\right)}{L_{2}}\right), \frac{1}{\mathfrak{g}_{0}^{s}} \varphi_{\varrho_{2}}\left(\frac{(1-b)\left(1-c_{4}\right)}{L_{4}}\right)\right\}, \\
& E=\min \left\{\frac{1}{\mathfrak{f}_{0}^{s}} \varphi_{\varrho_{1}}\left(\frac{b}{L_{1}}\right), \frac{1}{\mathfrak{f}_{0}^{s}} \varphi_{\varrho_{1}}\left(\frac{1-b}{L_{3}}\right)\right\},
\end{aligned}
$$




$$
F=\min \left\{\frac{1}{\mathfrak{g}_{0}^{s}} \varphi_{\varrho_{2}}\left(\frac{b}{L_{2}}\right), \frac{1}{\mathfrak{g}_{0}^{s}} \varphi_{\varrho_{2}}\left(\frac{1-b}{L_{4}}\right)\right\},
$$

where $\zeta_{1}=\theta_{1}^{\beta_{1}-1}, \zeta_{2}=\theta_{1}^{\beta_{2}-1}, \zeta=\min \left\{\zeta_{1}, \zeta_{2}\right\}$

Theorem 3.1 Assume that (I1) and (I2) hold, $\left[\theta_{1}, \theta_{2}\right] \subset[0,1]$ with $0<\theta_{1}<\theta_{2} \leq 1, c_{1}, c_{2} \in$ $[0,1], c_{3}, c_{4} \in(0,1), a \in[0,1]$ and $b \in(0,1)$.

(1) If $\mathfrak{f}_{0}^{s}, \mathfrak{g}_{0}^{s}, \mathfrak{f}_{\infty}^{i}, \mathfrak{g}_{\infty}^{i} \in(0, \infty), A<B$ and $C<D$, then, for each $\lambda \in(A, B)$ and $\mu \in(C, D)$, the problem (1), (2) has at least one positive solution $(x(t), y(t)), t \in[0,1]$.

(2) If $\mathfrak{f}_{0}^{s}=0, \mathfrak{g}_{0}^{s}, \mathfrak{f}_{\infty}^{i}, \mathfrak{g}_{\infty}^{i} \in(0, \infty)$ and $C<F$, then, for each $\lambda \in(A, \infty)$ and $\mu \in(C, F)$, the problem (1), (2) has at least one positive solution $(x(t), y(t)), t \in[0,1]$.

(3) If $\mathfrak{g}_{0}^{s}=0, \mathfrak{f}_{0}^{s}, \mathfrak{f}_{\infty}^{i}, \mathfrak{g}_{\infty}^{i} \in(0, \infty)$ and $A<E$, then, for each $\lambda \in(A, E)$ and $\mu \in(C, \infty)$, the problem (1), (2) has at least one positive solution $(x(t), y(t)), t \in[0,1]$.

(4) If $\mathfrak{f}_{0}^{s}=\mathfrak{g}_{0}^{s}=0, \mathfrak{f}_{\infty}^{i}, \mathfrak{g}_{\infty}^{i} \in(0, \infty)$, then, for each $\lambda \in(A, \infty)$ and $\mu \in(C, \infty)$, the problem (1), (2) has at least one positive solution $(x(t), y(t)), t \in[0,1]$.

(5) If $\mathfrak{f}_{0}^{s}, \mathfrak{g}_{0}^{s} \in(0, \infty)$ and at least one of $\mathfrak{f}_{\infty}^{i}, \mathfrak{g}_{\infty}^{i}$ is $\infty$, then, for each $\lambda \in(0, B)$ and $\mu \in(0, D)$, the problem (1), (2) has at least one positive solution $(x(t), y(t)), t \in[0,1]$.

(6) If $\mathfrak{f}_{0}^{s}=0, \mathfrak{g}_{0}^{s} \in(0, \infty)$ and at least one of $\mathfrak{f}_{\infty}^{i}, \mathfrak{g}_{\infty}^{i}$ is $\infty$, then, for each $\lambda \in(0, \infty)$ and $\mu \in(0, F)$, the problem (1), (2) has at least one positive solution $(x(t), y(t)), t \in[0,1]$.

(7) If $\mathfrak{f}_{0}^{s} \in(0, \infty), \mathfrak{g}_{0}^{s}=0$ and at least one of $\mathfrak{f}_{\infty}^{i}, \mathfrak{g}_{\infty}^{i}$ is $\infty$, then, for each $\lambda \in(0, E)$ and $\mu \in(0, \infty)$, the problem (1), (2) has at least one positive solution $(x(t), y(t)), t \in[0,1]$.

(8) If $\mathfrak{f}_{0}^{s}=\mathfrak{g}_{0}^{s}=0$ and at least one of $\mathfrak{f}_{\infty}^{i}, \mathfrak{g}_{\infty}^{i}$ is $\infty$, then, for each $\lambda \in(0, \infty)$ and $\mu \in(0, \infty)$, the problem (1), (2) has at least one positive solution $(x(t), y(t)), t \in[0,1]$.

Proof We consider the cone $\mathcal{P} \subset \mathcal{Y}$ and the operators $\mathcal{A}_{1}, \mathcal{A}_{2}$ and $\mathcal{A}$. The proofs of the above cases are similar, and for this reason we will prove only two cases, namely (1) and (6).

Case (1). We have $\mathfrak{f}_{0}^{s}, \mathfrak{g}_{0}^{s}, \mathfrak{f}_{\infty}^{i}, \mathfrak{g}_{\infty}^{i} \in(0, \infty), A<B$ and $C<D$. Let $\lambda \in(A, B)$ and $\mu \in(C, D)$. We consider $\epsilon>0$ such that $\epsilon<\mathfrak{f}_{\infty}^{i}, \epsilon<\mathfrak{g}_{\infty}^{i}$ and

$$
\begin{aligned}
& \max \left\{\frac{1}{\mathfrak{f}_{\infty}^{i}-\epsilon} \varphi_{\varrho_{1}}\left(\frac{a c_{1}}{\zeta \zeta_{1} \widetilde{L}_{1}}\right), \frac{1}{\mathfrak{f}_{\infty}^{i}-\epsilon} \varphi_{\varrho_{1}}\left(\frac{(1-a) c_{2}}{\zeta \widetilde{L}_{2}}\right)\right\} \\
& \quad \leq \lambda \leq \min \left\{\frac{1}{\mathfrak{f}_{0}^{s}+\epsilon} \varphi_{\varrho_{1}}\left(\frac{b c_{3}}{L_{1}}\right), \frac{1}{\mathfrak{f}_{0}^{s}+\epsilon} \varphi_{\varrho_{1}}\left(\frac{(1-b) c_{4}}{L_{3}}\right)\right\} \\
& \max \left\{\frac{1}{\mathfrak{g}_{\infty}^{i}-\epsilon} \varphi_{\varrho_{2}}\left(\frac{a\left(1-c_{1}\right)}{\zeta \zeta_{1} \widetilde{L}_{2}}\right), \frac{1}{\mathfrak{g}_{\infty}^{i}-\epsilon} \varphi_{\varrho_{2}}\left(\frac{(1-a)\left(1-c_{2}\right)}{\zeta \zeta_{2} \widetilde{L}_{4}}\right)\right\} \\
& \quad \leq \mu \leq \min \left\{\frac{1}{\mathfrak{g}_{0}^{s}+\epsilon} \varphi_{\varrho_{2}}\left(\frac{b\left(1-c_{3}\right)}{L_{2}}\right), \frac{1}{\mathfrak{g}_{0}^{s}+\epsilon} \varphi_{\varrho_{2}}\left(\frac{(1-b)\left(1-c_{4}\right)}{L_{4}}\right)\right\} .
\end{aligned}
$$

By using (I2) and the definitions of $\mathfrak{f}_{0}^{s}$ and $\mathfrak{g}_{0}^{s}$, we find that there exists $r_{1}>0$ such that

$$
f(t, x, y) \leq\left(\mathfrak{f}_{0}^{s}+\epsilon\right) \varphi_{\varrho_{1}}(x+y), \quad g(t, x, y) \leq\left(\mathfrak{g}_{0}^{s}+\epsilon\right) \varphi_{\varrho_{2}}(x+y)
$$

for all $t \in[0,1]$ and $x, y \geq 0, x+y \leq r_{1}$.

We introduce the set $\Lambda_{1}=\left\{(x, y) \in \mathcal{Y},\|(x, y)\|_{\mathcal{Y}}<r_{1}\right\}$. Let $(x, y) \in \mathcal{P} \cap \partial \Lambda_{1}$, that is $(x, y) \in \mathcal{P}$ with $\|(x, y)\| \mathcal{Y}=r_{1}$ or $\|x\|+\|y\|=r_{1}$. Then $x(t)+y(t) \leq r_{1}$ for all $t \in[0,1]$, and by 
Lemma 2.5, we deduce

$$
\begin{aligned}
& \mathcal{A}_{1}(x, y)(t) \\
& \leq \lambda^{\rho_{1}-1} \int_{0}^{1} \mathcal{J}_{1}(v) \varphi_{\rho_{1}}\left(\frac{1}{\Gamma\left(\alpha_{1}\right)} \int_{0}^{v}(v-s)^{\alpha_{1}-1} f(s, x(s), y(s)) d s\right) d v \\
& +\mu^{\rho_{2}-1} \int_{0}^{1} \mathcal{J}_{2}(v) \varphi_{\rho_{2}}\left(\frac{1}{\Gamma\left(\alpha_{2}\right)} \int_{0}^{v}(v-s)^{\alpha_{2}-1} g(s, x(s), y(s)) d s\right) d v \\
& \leq \lambda^{\rho_{1}-1} \int_{0}^{1} \mathcal{J}_{1}(v) \varphi_{\rho_{1}}\left(\frac{1}{\Gamma\left(\alpha_{1}\right)} \int_{0}^{v}(v-s)^{\alpha_{1}-1}\left(\mathfrak{f}_{0}^{s}+\epsilon\right) \varphi_{\varrho_{1}}(x(s)+y(s)) d s\right) d v \\
& +\mu^{\rho_{2}-1} \int_{0}^{1} \mathcal{J}_{2}(v) \varphi_{\rho_{2}}\left(\frac{1}{\Gamma\left(\alpha_{2}\right)} \int_{0}^{v}(v-s)^{\alpha_{2}-1}\left(\mathfrak{g}_{0}^{s}+\epsilon\right) \varphi_{\varrho_{2}}(x(s)+y(s)) d s\right) d v \\
& \leq \lambda^{\rho_{1}-1} \varphi_{\rho_{1}}\left(\mathfrak{f}_{0}^{s}+\epsilon\right) \int_{0}^{1} \mathcal{J}_{1}(v) \varphi_{\rho_{1}}\left(\frac{1}{\Gamma\left(\alpha_{1}\right)} \int_{0}^{v}(v-s)^{\alpha_{1}-1} \varphi_{\varrho_{1}}(\|x\|+\|y\|) d s\right) d v \\
& +\mu^{\rho_{2}-1} \varphi_{\rho_{2}}\left(\mathfrak{g}_{0}^{s}+\epsilon\right) \int_{0}^{1} \mathcal{J}_{2}(v) \varphi_{\rho_{2}}\left(\frac{1}{\Gamma\left(\alpha_{2}\right)} \int_{0}^{v}(v-s)^{\alpha_{2}-1} \varphi_{\varrho_{2}}(\|x\|+\|y\|) d s\right) d \nu \\
& =\lambda^{\rho_{1}-1} \varphi_{\rho_{1}}\left(\mathfrak{f}_{0}^{s}+\epsilon\right)\|(x, y)\|_{\mathcal{Y}} \int_{0}^{1} \mathcal{J}_{1}(\nu) \frac{1}{\left(\Gamma\left(\alpha_{1}+1\right)\right)^{\rho_{1}-1}} v^{\alpha_{1}\left(\rho_{1}-1\right)} d \nu \\
& +\mu^{\rho_{2}-1} \varphi_{\rho_{2}}\left(\mathfrak{g}_{0}^{s}+\epsilon\right)\|(x, y)\|_{\mathcal{Y}} \int_{0}^{1} \mathcal{J}_{2}(\nu) \frac{1}{\left(\Gamma\left(\alpha_{2}+1\right)\right)^{\rho_{2}-1}} v^{\alpha_{2}\left(\rho_{2}-1\right)} d \nu \\
& =\left[\varphi_{\rho_{1}}\left(\lambda\left(\mathfrak{f}_{0}^{s}+\epsilon\right)\right) L_{1}+\varphi_{\rho_{2}}\left(\mu\left(\mathfrak{g}_{0}^{s}+\epsilon\right)\right) L_{2}\right]\|(x, y)\|_{\mathcal{Y}} \\
& \leq\left[b c_{3}+b\left(1-c_{3}\right)\right]\|(x, y)\|_{\mathcal{Y}}=b\|(x, y)\|_{\mathcal{Y}}, \quad \forall t \in[0,1] \text {. }
\end{aligned}
$$

Therefore $\left\|\mathcal{A}_{1}(x, y)\right\| \leq b\|(x, y)\| \mathcal{Y}$.

In a similar manner we obtain

$$
\begin{aligned}
\mathcal{A}_{2}(x, y)(t) \leq & \lambda^{\rho_{1}-1} \varphi_{\rho_{1}}\left(\mathfrak{f}_{0}^{s}+\epsilon\right)\|(x, y)\| \mathcal{Y} \int_{0}^{1} \mathcal{J}_{3}(v) \frac{1}{\left(\Gamma\left(\alpha_{1}+1\right)\right)^{\rho_{1}-1}} v^{\alpha_{1}\left(\rho_{1}-1\right)} d v \\
& +\mu^{\rho_{2}-1} \varphi_{\rho_{2}}\left(\mathfrak{g}_{0}^{s}+\epsilon\right)\|(x, y)\|_{\mathcal{Y}} \int_{0}^{1} \mathcal{J}_{4}(v) \frac{1}{\left(\Gamma\left(\alpha_{2}+1\right)\right)^{\rho_{2}-1}} v^{\alpha_{2}\left(\rho_{2}-1\right)} d v \\
= & {\left[\varphi_{\rho_{1}}\left(\lambda\left(\mathfrak{f}_{0}^{s}+\epsilon\right)\right) L_{3}+\varphi_{\rho_{2}}\left(\mu\left(\mathfrak{g}_{0}^{s}+\epsilon\right)\right) L_{4}\right]\|(x, y)\|_{\mathcal{Y}} } \\
\leq & {\left[(1-b) c_{4}+(1-b)\left(1-c_{4}\right)\right]\|(x, y)\|_{\mathcal{Y}}=(1-b)\|(x, y)\|_{\mathcal{Y}}, \quad \forall t \in[0,1] . }
\end{aligned}
$$

Then $\left\|\mathcal{A}_{2}(x, y)\right\| \leq(1-b)\|(x, y)\| \mathcal{Y}$.

Therefore, for $(x, y) \in \mathcal{P} \cap \partial \Lambda_{1}$, we conclude

$$
\|\mathcal{A}(x, y)\|_{\mathcal{Y}}=\left\|\mathcal{A}_{1}(x, y)\right\|+\left\|\mathcal{A}_{2}(x, y)\right\| \leq b\|(x, y)\|_{\mathcal{Y}}+(1-b)\|(x, y)\|_{\mathcal{Y}}=\|(x, y)\|_{\mathcal{Y}} .
$$

By the definitions of $\mathfrak{f}_{\infty}^{i}$ and $\mathfrak{g}_{\infty}^{i}$, we find that there exists $r_{2}^{\prime}>0$ such that

$$
f(t, x, y) \geq\left(\mathfrak{f}_{\infty}^{i}-\epsilon\right) \varphi_{\varrho_{1}}(x+y), \quad g(t, x, y) \geq\left(\mathfrak{g}_{\infty}^{i}-\epsilon\right) \varphi_{\varrho_{2}}(x+y),
$$

for all $t \in\left[\theta_{1}, \theta_{2}\right]$ and $x, y \geq 0, x+y \geq r_{2}^{\prime}$. 
We consider $r_{2}=\max \left\{2 r_{1}, r_{2}^{\prime} / \zeta\right\}$ and we introduce the set $\Lambda_{2}=\left\{(x, y) \in \mathcal{Y},\|(x, y)\| \mathcal{Y}<r_{2}\right\}$.

Then, for $(x, y) \in \mathcal{P} \cap \partial \Lambda_{2}$, we deduce

$$
\begin{aligned}
x(t)+y(t) & \geq \min _{t \in\left[\theta_{1}, \theta_{2}\right]} t^{\beta_{1}-1}\|x\|+\min _{t \in\left[\theta_{1}, \theta_{2}\right]} t^{\beta_{2}-1}\|y\|=\theta_{1}^{\beta_{1}-1}\|x\|+\theta_{1}^{\beta_{2}-1}\|y\| \\
& =\zeta_{1}\|x\|+\zeta_{2}\|y\| \geq \zeta\|(x, y)\|_{\mathcal{Y}}=\zeta r_{2} \geq r_{2}^{\prime}, \quad \forall t \in\left[\theta_{1}, \theta_{2}\right] .
\end{aligned}
$$

Therefore, by Lemma 2.5, we obtain

$$
\begin{aligned}
\mathcal{A}_{1}(x, y)\left(\theta_{1}\right) & \\
\geq & \lambda^{\rho_{1}-1} \int_{0}^{1} \theta_{1}^{\beta_{1}-1} \mathcal{J}_{1}(v) \varphi_{\rho_{1}}\left(I_{0_{1}}^{\alpha_{1}} f(v, x(v), y(v))\right) d v \\
& +\mu^{\rho_{2}-1} \int_{0}^{1} \theta_{1}^{\beta_{1}-1} \mathcal{J}_{2}(v) \varphi_{\rho_{2}}\left(I_{0+}^{\alpha_{2}} g(v, x(v), y(v))\right) d v \\
\geq & \lambda^{\rho_{1}-1} \theta_{1}^{\beta_{1}-1} \int_{\theta_{1}}^{\theta_{2}} \mathcal{J}_{1}(v) \varphi_{\rho_{1}}\left(\frac{1}{\Gamma\left(\alpha_{1}\right)} \int_{\theta_{1}}^{v}(v-s)^{\alpha_{1}-1} f(s, x(s), y(s)) d s\right) d v \\
& +\mu^{\rho_{2}-1} \theta_{1}^{\beta_{1}-1} \int_{\theta_{1}}^{\theta_{2}} \mathcal{J}_{2}(v) \varphi_{\rho_{2}}\left(\frac{1}{\Gamma\left(\alpha_{2}\right)} \int_{\theta_{1}}^{v}(v-s)^{\alpha_{2}-1} g(s, x(s), y(s)) d s\right) d v \\
\geq & \lambda^{\rho_{1}-1} \theta_{1}^{\beta_{1}-1} \int_{\theta_{1}}^{\theta_{2}} \mathcal{J}_{1}(v) \varphi_{\rho_{1}}\left(\frac{1}{\Gamma\left(\alpha_{1}\right)} \int_{\theta_{1}}^{v}(v-s)^{\alpha_{1}-1}\left(\mathfrak{f}_{\infty}^{i}-\epsilon\right) \varphi_{\varrho_{1}}(x(s)+y(s)) d s\right) d v \\
& +\mu^{\rho_{2}-1} \theta_{1}^{\beta_{1}-1} \int_{\theta_{1}}^{\theta_{2}} \mathcal{J}_{2}(v) \varphi_{\rho_{2}}\left(\frac{1}{\Gamma\left(\alpha_{2}\right)} \int_{\theta_{1}}^{v}(v-s)^{\alpha_{2}-1}\left(\mathfrak{g}_{\infty}^{i}-\epsilon\right) \varphi_{\varrho_{1}}(x(s)+y(s)) d s\right) d v \\
\geq & \lambda^{\rho_{1}-1} \theta_{1}^{\beta_{1}-1} \int_{\theta_{1}}^{\theta_{2}} \mathcal{J}_{1}(v) \varphi_{\rho_{1}}\left(\frac{1}{\Gamma\left(\alpha_{1}\right)} \int_{\theta_{1}}^{v}(v-s)^{\alpha_{1}-1}\left(\mathfrak{f}_{\infty}^{i}-\epsilon\right) \varphi_{\varrho_{1}}\left(\zeta\|(x, y)\|_{\mathcal{Y}}\right) d s\right) d v \\
& +\mu^{\rho_{2}-1} \theta_{1}^{\beta_{1}-1} \int_{\theta_{1}}^{\theta_{2}} \mathcal{J}_{2}(v) \varphi_{\rho_{2}}\left(\frac{1}{\Gamma\left(\alpha_{2}\right)} \int_{\theta_{1}}^{v}(v-s)^{\alpha_{2}-1}\left(\mathfrak{g}_{\infty}^{i}-\epsilon\right) \varphi_{\varrho_{1}}\left(\zeta\|(x, y)\|_{\mathcal{Y}}\right) d s\right) d v \\
= & \zeta \zeta_{1} \varphi_{\rho_{1}}\left(\lambda\left(\mathfrak{f}_{\infty}^{i}-\epsilon\right)\right)\|(x, y)\|_{\mathcal{Y}} \int_{\theta_{1}}^{\theta_{2}} \mathcal{J}_{1}(v) \frac{1}{\left(\Gamma\left(\alpha_{1}+1\right)\right)^{\rho_{1}-1}}\left(v-\theta_{1}\right)^{\alpha_{1}\left(\rho_{1}-1\right)} d v \\
& +\zeta \zeta_{1} \varphi_{\rho_{2}}\left(\mu\left(\mathfrak{g}_{\infty}^{i}-\epsilon\right)\right)\|(x, y)\|_{\mathcal{Y}} \int_{\theta_{1}}^{\theta_{2}} \mathcal{J}_{2}(v) \frac{1}{\left(\Gamma\left(\alpha_{2}+1\right)\right)^{\rho_{2}-1}}\left(v-\theta_{1}\right)^{\alpha_{2}\left(\rho_{2}-1\right)} d v \\
& \left.\zeta \zeta_{1} \varphi_{\rho_{1}}\left(\lambda\left(\mathfrak{f}_{\infty}^{i}-\epsilon\right)\right) \widetilde{L}_{1}+\zeta \zeta_{1} \varphi_{\rho_{2}}\left(\mu\left(\mathfrak{g}_{\infty}^{i}-\epsilon\right)\right) \widetilde{L}_{2}\right]\|(x, y)\|_{\mathcal{Y}} \\
\geq & \left.\left.c_{1}\right)\right]\|(x, y)\|_{\mathcal{Y}}=a\|(x, y)\|_{\mathcal{Y}} \cdot
\end{aligned}
$$

Then $\left\|\mathcal{A}_{1}(x, y)\right\| \geq \mathcal{A}_{1}(x, y)\left(\theta_{1}\right) \geq a\|(x, y)\|_{\mathcal{Y}}$.

In a similar manner, we deduce

$$
\begin{aligned}
& \mathcal{A}_{2}(x, y)\left(\theta_{1}\right) \\
& \geq \zeta \zeta_{2} \varphi_{\rho_{1}}\left(\lambda\left(\mathfrak{f}_{\infty}^{i}-\epsilon\right)\right)\|(x, y)\|_{\mathcal{Y}} \int_{\theta_{1}}^{\theta_{2}} \mathcal{J}_{3}(v) \frac{1}{\left(\Gamma\left(\alpha_{1}+1\right)\right)^{\rho_{1}-1}}\left(v-\theta_{1}\right)^{\alpha_{1}\left(\rho_{1}-1\right)} d v \\
& \quad+\zeta \zeta_{2} \varphi_{\rho_{2}}\left(\mu\left(\mathfrak{g}_{\infty}^{i}-\epsilon\right)\right)\|(x, y)\|_{\mathcal{Y}} \int_{\theta_{1}}^{\theta_{2}} \mathcal{J}_{4}(v) \frac{1}{\left(\Gamma\left(\alpha_{2}+1\right)\right)^{\rho_{2}-1}}\left(v-\theta_{1}\right)^{\alpha_{2}\left(\rho_{2}-1\right)} d v
\end{aligned}
$$




$$
\begin{aligned}
& =\left[\zeta \zeta_{2} \varphi_{\rho_{1}}\left(\lambda\left(\mathfrak{f}_{\infty}^{i}-\epsilon\right)\right) \widetilde{L}_{3}+\zeta \zeta_{2} \varphi_{\rho_{2}}\left(\mu\left(\mathfrak{g}_{\infty}^{i}-\epsilon\right)\right) \widetilde{L}_{4}\right]\|(x, y)\|_{\mathcal{Y}} \\
& \geq\left[(1-a) c_{2}+(1-a)\left(1-c_{2}\right)\right]\|(x, y)\|_{\mathcal{Y}}=(1-a)\|(x, y)\|_{\mathcal{Y}} .
\end{aligned}
$$

Hence $\left\|\mathcal{A}_{2}(x, y)\right\| \geq \mathcal{A}_{2}(x, y)\left(\theta_{1}\right) \geq(1-a)\|(x, y)\| \mathcal{Y}$.

Therefore for $(x, y) \in \mathcal{P} \cap \partial \Lambda_{2}$ we find

$$
\|\mathcal{A}(x, y)\|_{\mathcal{Y}}=\left\|\mathcal{A}_{1}(x, y)\right\|+\left\|\mathcal{A}_{2}(x, y)\right\| \geq a\|(x, y)\|_{\mathcal{Y}}+(1-a)\|(x, y)\|_{\mathcal{Y}}=\|(x, y)\|_{\mathcal{Y}}
$$

By Lemma 3.1, Eqs. (20), (21) and the Guo-Krasnosel'skii fixed point theorem, we conclude that $\mathcal{A}$ has a fixed point $(x, y) \in \mathcal{P} \cap\left(\bar{\Lambda}_{2} \backslash \Lambda_{1}\right)$ such that $r_{1} \leq\|x\|+\|y\| \leq r_{2}$, $x(t) \geq t^{\beta_{1}-1}\|x\|, y(t) \geq t^{\beta_{2}-1}\|y\|$ for all $t \in[0,1]$. If $\|x\|>0$ then $x(t)>0$ for all $t \in(0,1]$ and if $\|y\|>0$ then $y(t)>0$ for all $t \in(0,1]$. Therefore $(x, y)$ is a positive solution for problem (1), (2).

Case (6). We have $\mathfrak{f}_{0}^{s}=0, \mathfrak{g}_{0}^{s} \in(0, \infty)$ and $\mathfrak{f}_{\infty}^{i}=\infty$. Let $\lambda \in(0, \infty)$ and $\mu \in(0, F)$. We choose $\widetilde{c}_{3} \in\left(0,1-\varphi_{\rho_{2}}\left(\mu \mathfrak{g}_{0}^{s}\right) \frac{L_{2}}{b}\right)$ and $\widetilde{c}_{4} \in\left(0,1-\varphi_{\rho_{2}}\left(\mu \mathfrak{g}_{0}^{s}\right) \frac{L_{4}}{1-b}\right)$. The choice of $\widetilde{c}_{3}$ and $\widetilde{c}_{4}$ is possible because $\mu<\frac{1}{\mathfrak{g}_{0}^{s}} \varphi_{\varrho_{2}}\left(\frac{b}{L_{2}}\right)$ and $\mu<\frac{1}{\mathfrak{g}_{0}^{s}} \varphi_{\varrho_{2}}\left(\frac{1-b}{L_{4}}\right)$. Let $\epsilon>0$ be such that

$$
\begin{aligned}
& \epsilon \varphi_{\varrho_{1}}\left(\frac{1}{\zeta \zeta_{1} \widetilde{L}_{1}}\right) \leq \lambda \leq \min \left\{\frac{1}{\epsilon} \varphi_{\varrho_{1}}\left(\frac{b \widetilde{c}_{3}}{L_{1}}\right), \frac{1}{\epsilon} \varphi_{\varrho_{1}}\left(\frac{(1-b) \widetilde{c}_{4}}{L_{3}}\right)\right\}, \\
& \mu \leq \min \left\{\frac{1}{\mathfrak{g}_{0}^{s}+\epsilon} \varphi_{\varrho_{2}}\left(\frac{b\left(1-\widetilde{c}_{3}\right)}{L_{2}}\right), \frac{1}{\mathfrak{g}_{0}^{s}+\epsilon}\left(\frac{(1-b)\left(1-\widetilde{c}_{4}\right)}{L_{4}}\right)\right\} .
\end{aligned}
$$

By using (I2) and the definitions of $\mathfrak{f}_{0}^{s}$ and $\mathfrak{g}_{0}^{s}$, we find that there exists $r_{1}>0$ such that

$$
f(t, x, y) \leq \epsilon \varphi_{\varrho_{1}}(x+y), \quad g(t, x, y) \leq\left(\mathfrak{g}_{0}^{s}+\epsilon\right) \varphi_{\varrho_{2}}(x+y)
$$

for all $t \in[0,1]$ and $x, y \geq 0, x+y \leq r_{1}$.

We introduce the set $\Lambda_{1}=\left\{(x, y) \in \mathcal{Y},\|(x, y)\|_{\mathcal{Y}}<r_{1}\right\}$. As in the proof of Case (1), for any $(x, y) \in \mathcal{P} \cap \partial \Lambda_{1}$, we deduce

$$
\begin{aligned}
\mathcal{A}_{1}(x, y)(t) & \leq\left[\varphi_{\rho_{1}}(\lambda \epsilon) L_{1}+\varphi_{\rho_{2}}\left(\mu\left(\mathfrak{g}_{0}^{s}+\epsilon\right)\right) L_{2}\right]\|(x, y)\|_{\mathcal{Y}} \\
& \leq\left[b \widetilde{c}_{3}+b\left(1-\widetilde{c}_{3}\right)\right]\|(x, y)\|_{\mathcal{Y}}=b\|(x, y)\|_{\mathcal{Y}}, \quad \forall t \in[0,1], \\
\mathcal{A}_{2}(x, y)(t) & \leq\left[\varphi_{\rho_{1}}(\lambda \epsilon) L_{3}+\varphi_{\rho_{2}}\left(\mu\left(\mathfrak{g}_{0}^{s}+\epsilon\right)\right) L_{4}\right]\|(x, y)\|_{\mathcal{Y}} \\
& \leq\left[(1-b) \widetilde{c}_{4}+(1-b)\left(1-\widetilde{c}_{4}\right)\right]\|(x, y)\|_{\mathcal{Y}}=(1-b)\|(x, y)\|_{\mathcal{Y}}, \quad \forall t \in[0,1],
\end{aligned}
$$

and so $\|\mathcal{A}(x, y)\|_{\mathcal{Y}} \leq\|(x, y)\|_{\mathcal{Y}}$.

For the second part, by the definition of $\mathfrak{f}_{\infty}^{i}$, we find that there exists $r_{2}^{\prime}>0$ such that

$$
f(t, x, y) \geq \frac{1}{\epsilon} \varphi_{\varrho_{1}}(x+y), \quad \forall t \in\left[\theta_{1}, \theta_{2}\right], x, y \geq 0, x+y \geq r_{2}^{\prime} .
$$

We consider $r_{2}=\max \left\{2 r_{1}, r_{2}^{\prime} / \zeta\right\}$ and we introduce the set $\Lambda_{2}=\left\{(x, y) \in \mathcal{Y},\|(x, y)\| \mathcal{Y}<r_{2}\right\}$. Then, for any $(x, y) \in \mathcal{P} \cap \partial \Lambda_{2}$, we obtain as in Case (1) that $x(t)+y(t) \geq \zeta\|(x, y)\| \mathcal{Y}=\zeta r_{2} \geq$ $r_{2}^{\prime}$ for all $t \in\left[\theta_{1}, \theta_{2}\right]$. 
Hence by Lemma 2.5 we deduce

$$
\begin{aligned}
\mathcal{A}_{1}(x, y)\left(\theta_{1}\right) & \\
\geq & \lambda^{\rho_{1}-1} \int_{0}^{1} \theta_{1}^{\beta_{1}-1} \mathcal{J}_{1}(v) \varphi_{\rho_{1}}\left(I_{0+}^{\alpha_{1}} f(v, x(v), y(v))\right) d v \\
& +\mu^{\rho_{2}-1} \int_{0}^{1} \theta_{1}^{\beta_{1}-1} \mathcal{J}_{2}(v) \varphi_{\rho_{2}}\left(I_{0+}^{\alpha_{2}} g(v, x(v), y(v))\right) d v \\
\geq & \lambda^{\rho_{1}-1} \int_{0}^{1} \theta_{1}^{\beta_{1}-1} \mathcal{J}_{1}(v) \varphi_{\rho_{1}}\left(I_{0+}^{\alpha_{1}} f(v, x(v), y(v))\right) d v \\
\geq & \lambda^{\rho_{1}-1} \theta_{1}^{\beta_{1}-1} \int_{\theta_{1}}^{\theta_{2}} \mathcal{J}_{1}(v) \varphi_{\rho_{1}}\left(\frac{1}{\Gamma\left(\alpha_{1}\right)} \int_{\theta_{1}}^{v}(v-s)^{\alpha_{1}-1} f(s, x(s), y(s)) d s\right) d v \\
\geq & \lambda^{\rho_{1}-1} \theta_{1}^{\beta_{1}-1} \int_{\theta_{1}}^{\theta_{2}} \mathcal{J}_{1}(v) \varphi_{\rho_{1}}\left(\frac{1}{\Gamma\left(\alpha_{1}\right)} \int_{\theta_{1}}^{v}(v-s)^{\alpha_{1}-1} \frac{1}{\epsilon} \varphi_{\varrho_{1}}(x(s)+y(s)) d s\right) d v \\
\geq & \lambda^{\rho_{1}-1} \theta_{1}^{\beta_{1}-1} \int_{\theta_{1}}^{\theta_{2}} \mathcal{J}_{1}(v) \varphi_{\rho_{1}}\left(\frac{1}{\Gamma\left(\alpha_{1}\right)} \int_{\theta_{1}}^{v}(v-s)^{\alpha_{1}-1} \frac{1}{\epsilon} \varphi_{\varrho_{1}}\left(\zeta\|(x, y)\|_{\mathcal{Y}}\right) d s\right) d v \\
= & \zeta \zeta_{1} \varphi_{\rho_{1}}\left(\frac{\lambda}{\epsilon}\right)\|(x, y)\| \mathcal{Y} \int_{\theta_{1}}^{\theta_{2}} \mathcal{J}_{1}(v) \frac{1}{\left(\Gamma\left(\alpha_{1}+1\right)\right)^{\rho_{1}-1}}\left(v-\theta_{1}\right)^{\alpha_{1}\left(\rho_{1}-1\right)} d v \\
= & \zeta \zeta_{1} \varphi_{\rho_{1}}\left(\frac{\lambda}{\epsilon}\right)\|(x, y)\| \widetilde{\mathcal{L}}_{1} \geq\|(x, y)\|_{\mathcal{Y}} \cdot
\end{aligned}
$$

Then we conclude that $\left\|\mathcal{A}_{1}(x, y)\right\| \geq \mathcal{A}_{1}(x, y)\left(\theta_{1}\right) \geq\|(x, y)\| \mathcal{Y}$ and $\|\mathcal{A}(x, y)\| \mathcal{Y} \geq\left\|\mathcal{A}_{1}(x, y)\right\| \geq$ $\|(x, y)\| \mathcal{y}$. Therefore we obtain the conclusion of the theorem.

Next for $\mathfrak{f}_{0}^{i}, \mathfrak{g}_{0}^{i}, \mathfrak{f}_{\infty}^{s}, \mathfrak{g}_{\infty}^{s} \in(0, \infty)$ and numbers $c_{1}, c_{2} \in[0,1], c_{3}, c_{4} \in(0,1), a \in[0,1]$ and $b \in(0,1)$, we introduce the numbers

$$
\begin{aligned}
& \widetilde{A}=\max \left\{\frac{1}{\mathfrak{f}_{0}^{i}} \varphi_{\varrho_{1}}\left(\frac{a c_{1}}{\zeta \zeta_{1} \widetilde{L}_{1}}\right), \frac{1}{\mathfrak{f}_{0}^{i}} \varphi_{\varrho_{1}}\left(\frac{(1-a) c_{2}}{\zeta \zeta_{2} \widetilde{L}_{3}}\right)\right\}, \\
& \widetilde{B}=\min \left\{\frac{1}{\mathfrak{f}_{\infty}^{s}} \varphi_{\varrho_{1}}\left(\frac{b c_{3}}{L_{1}}\right), \frac{1}{\mathfrak{f}_{\infty}^{s}} \varphi_{\varrho_{1}}\left(\frac{(1-b) c_{4}}{L_{3}}\right)\right\}, \\
& \widetilde{C}=\max \left\{\frac{1}{\mathfrak{g}_{0}^{i}} \varphi_{\varrho_{2}}\left(\frac{a\left(1-c_{1}\right)}{\zeta \zeta_{1} \widetilde{L}_{2}}\right), \frac{1}{\mathfrak{g}_{0}^{i}} \varphi_{\varrho_{2}}\left(\frac{(1-a)\left(1-c_{2}\right)}{\zeta \zeta_{2} \widetilde{L}_{4}}\right)\right\}, \\
& \widetilde{E}=\min \left\{\frac{1}{\mathfrak{g}_{\infty}^{s}} \varphi_{\varrho_{2}}\left(\frac{b\left(1-c_{3}\right)}{L_{2}}\right), \frac{1}{\mathfrak{g}_{\infty}^{s}} \varphi_{\varrho_{2}}\left(\frac{(1-b)\left(1-c_{4}\right)}{L_{4}}\right)\right\}, \\
& \widetilde{F}=\min \left\{\frac{1}{\mathfrak{f}_{\infty}^{s}} \varphi_{\varrho_{1}}\left(\frac{b}{L_{1}}\right), \frac{1}{\mathfrak{f}_{\infty}^{s}} \varphi_{\varrho_{1}}\left(\frac{1-b}{L_{3}}\right)\right\}, \\
& \left.\mathfrak{g}_{\infty}^{s} \varphi_{\varrho_{2}}\left(\frac{b}{L_{2}}\right), \frac{1}{\mathfrak{g}_{\infty}^{s}} \varphi_{\varrho_{2}}\left(\frac{1-b}{L_{4}}\right)\right\} .
\end{aligned}
$$

Theorem 3.2 Assume that (I1) and (I2) hold, $\left[\theta_{1}, \theta_{2}\right] \subset[0,1]$ with $0<\theta_{1}<\theta_{2} \leq 1, c_{1}, c_{2} \in$ $[0,1], c_{3}, c_{4} \in(0,1), a \in[0,1]$ and $b \in(0,1)$.

(1) If $\mathfrak{f}_{0}^{i}, \mathfrak{g}_{0}^{i}, \mathfrak{f}_{\infty}^{s}, \mathfrak{g}_{\infty}^{s} \in(0, \infty), \widetilde{A}<\widetilde{B}$ and $\widetilde{C}<\widetilde{D}$, then, for each $\lambda \in(\widetilde{A}, \widetilde{B})$ and $\mu \in(\widetilde{C}, \widetilde{D})$, the problem (1), (2) has at least one positive solution $(x(t), y(t)), t \in[0,1]$.

(2) If $\mathfrak{f}_{0}^{i}, \mathfrak{g}_{0}^{i}, \mathfrak{f}_{\infty}^{s} \in(0, \infty), \mathfrak{g}_{\infty}^{s}=0$ and $\widetilde{A}<\widetilde{E}$, then, for each $\lambda \in(\widetilde{A}, \widetilde{E})$ and $\mu \in(\widetilde{C}, \infty)$, the problem (1), (2) has at least one positive solution $(x(t), y(t)), t \in[0,1]$. 
(3) If $\mathfrak{f}_{0}^{i}, \mathfrak{g}_{0}^{i}, \mathfrak{g}_{\infty}^{s} \in(0, \infty), \mathfrak{f}_{\infty}^{s}=0$ and $\widetilde{C}<\widetilde{F}$, then, for each $\lambda \in(\widetilde{A}, \infty)$ and $\mu \in(\widetilde{C}, \widetilde{F})$, the problem (1), (2) has at least one positive solution $(x(t), y(t)), t \in[0,1]$.

(4) If $\mathfrak{f}_{0}^{i}, \mathfrak{g}_{0}^{i} \in(0, \infty), \mathfrak{f}_{\infty}^{s}=\mathfrak{g}_{\infty}^{s}=0$, then, for each $\lambda \in(\widetilde{A}, \infty)$ and $\mu \in(\widetilde{C}, \infty)$, the problem (1), (2) has at least one positive solution $(x(t), y(t)), t \in[0,1]$.

(5) If $\mathfrak{f}_{\infty}^{s}, \mathfrak{g}_{\infty}^{s} \in(0, \infty)$ and at least one of $\mathfrak{f}_{0}^{i}, \mathfrak{g}_{0}^{i}$ is $\infty$, then, for each $\lambda \in(0, \widetilde{B})$ and $\mu \in(0, \widetilde{D})$, the problem (1), (2) has at least one positive solution $(x(t), y(t)), t \in[0,1]$.

(6) If $\mathfrak{f}_{\infty}^{s} \in(0, \infty), \mathfrak{g}_{\infty}^{s}=0$ and at least one of $\mathfrak{f}_{0}^{i}, \mathfrak{g}_{0}^{i}$ is $\infty$, then, for each $\lambda \in(0, \widetilde{E})$ and $\mu \in(0, \infty)$, the problem (1), (2) has at least one positive solution $(x(t), y(t)), t \in[0,1]$.

(7) If $\mathfrak{f}_{\infty}^{s}=0, \mathfrak{g}_{\infty}^{s} \in(0, \infty)$ and at least one of $\mathfrak{f}_{0}^{i}, \mathfrak{g}_{0}^{i}$ is $\infty$, then, for each $\lambda \in(0, \infty)$ and $\mu \in(0, \widetilde{F})$, the problem (1), (2) has at least one positive solution $(x(t), y(t)), t \in[0,1]$.

(8) If $\mathfrak{f}_{\infty}^{s}=\mathfrak{g}_{\infty}^{s}=0$ and at least one of $\mathfrak{f}_{0}^{i}, \mathfrak{g}_{0}^{i}$ is $\infty$, then, for each $\lambda \in(0, \infty)$ and $\mu \in(0, \infty)$, the problem (1), (2) has at least one positive solution $(x(t), y(t)), t \in[0,1]$.

Proof We consider again the cone $\mathcal{P} \subset \mathcal{Y}$ and the operators $\mathcal{A}_{1}, \mathcal{A}_{2}$ and $\mathcal{A}$. The proofs of the above cases are similar, and for this reason we will prove only two cases, namely (1) and (6).

Case (1). We have $\mathfrak{f}_{0}^{i}, \mathfrak{g}_{0}^{i}, \mathfrak{f}_{\infty}^{s}, \mathfrak{g}_{\infty}^{s} \in(0, \infty), \widetilde{A}<\widetilde{B}$ and $\widetilde{C}<\widetilde{D}$. Let $\lambda \in(\widetilde{A}, \widetilde{B})$ and $\mu \in(\widetilde{C}, \widetilde{D})$. We consider $\epsilon>0$ such that $\epsilon<\mathfrak{f}_{0}^{i}, \epsilon<\mathfrak{g}_{0}^{i}$ and

$$
\begin{aligned}
& \max \left\{\frac{1}{\mathfrak{f}_{0}^{i}-\epsilon} \varphi_{\varrho_{1}}\left(\frac{a c_{1}}{\zeta \zeta_{1} \widetilde{L}_{1}}\right), \frac{1}{\mathfrak{f}_{0}^{i}-\epsilon} \varphi_{\varrho_{1}}\left(\frac{(1-a) c_{2}}{\zeta \zeta_{2} \widetilde{L}_{3}}\right)\right\} \\
& \quad \leq \lambda \leq \min \left\{\frac{1}{\mathfrak{f}_{\infty}^{s}+\epsilon} \varphi_{\varrho_{1}}\left(\frac{b c_{3}}{L_{1}}\right), \frac{1}{\mathfrak{f}_{\infty}^{s}+\epsilon} \varphi_{\varrho_{1}}\left(\frac{(1-b) c_{4}}{L_{3}}\right)\right\}, \\
& \max \left\{\frac{1}{\mathfrak{g}_{0}^{i}-\epsilon} \varphi_{\varrho_{2}}\left(\frac{a\left(1-c_{1}\right)}{\zeta \widetilde{L}_{1}}\right), \frac{1}{\mathfrak{g}_{0}^{i}-\epsilon} \varphi_{\varrho_{2}}\left(\frac{(1-a)\left(1-c_{2}\right)}{\zeta \zeta_{2} \widetilde{L}_{4}}\right)\right\} \\
& \quad \leq \mu \leq \min \left\{\frac{1}{\mathfrak{g}_{\infty}^{s}+\epsilon} \varphi_{\varrho_{2}}\left(\frac{b\left(1-c_{3}\right)}{L_{2}}\right), \frac{1}{\mathfrak{g}_{\infty}^{s}+\epsilon}\left(\frac{(1-b)\left(1-c_{4}\right)}{L_{4}}\right)\right\} .
\end{aligned}
$$

By using (I2) and the definitions of $\mathfrak{f}_{0}^{i}$ and $\mathfrak{g}_{0}^{i}$ we deduce that there exists $r_{3}>0$ such that

$$
f(t, x, y) \geq\left(\mathfrak{f}_{0}^{i}-\epsilon\right) \varphi_{\varrho_{1}}(x+y), \quad g(t, x, y) \geq\left(\mathfrak{g}_{0}^{i}-\epsilon\right) \varphi_{\varrho_{2}}(x+y),
$$

for all $t \in\left[\theta_{1}, \theta_{2}\right], x, y \geq 0, x+y \leq r_{3}$.

We introduce the set $\Lambda_{3}=\left\{(x, y) \in \mathcal{Y},\|(x, y)\| \mathcal{Y}<r_{3}\right\}$. Let $(x, y) \in \mathcal{P} \cap \partial \Lambda_{3}$, that is $(x, y) \in$ $\mathcal{P}$ with $\|(x, y)\| \mathcal{Y}=r_{3}$ or $\|x\|+\|y\|=r_{3}$. Then $x(t)+y(t) \leq r_{3}$ for all $t \in[0,1]$, and $x(t)+y(t) \geq$ $\zeta\|(x, y)\| \mathcal{Y}$ for all $t \in\left[\theta_{1}, \theta_{2}\right]$ (see the proof of Case (1) in Theorem 3.1). So, by Lemma 2.5, we obtain

$$
\begin{aligned}
& \mathcal{A}_{1}(x, y)\left(\theta_{1}\right) \\
& \geq \lambda^{\rho_{1}-1} \theta_{1}^{\beta_{1}-1} \int_{\theta_{1}}^{\theta_{2}} \mathcal{J}_{1}(v) \varphi_{\rho_{1}}\left(\frac{1}{\Gamma\left(\alpha_{1}\right)} \int_{\theta_{1}}^{v}(v-s)^{\alpha_{1}-1} f(s, x(s), y(s)) d s\right) d v \\
& \quad+\mu^{\rho_{2}-1} \theta_{1}^{\beta_{1}-1} \int_{\theta_{1}}^{\theta_{2}} \mathcal{J}_{2}(v) \varphi_{\rho_{2}}\left(\frac{1}{\Gamma\left(\alpha_{2}\right)} \int_{\theta_{1}}^{v}(v-s)^{\alpha_{2}-1} g(s, x(s), y(s)) d s\right) d v \\
& \geq \lambda^{\rho_{1}-1} \theta_{1}^{\beta_{1}-1} \int_{\theta_{1}}^{\theta_{2}} \mathcal{J}_{1}(v) \varphi_{\rho_{1}}\left(\frac{1}{\Gamma\left(\alpha_{1}\right)} \int_{\theta_{1}}^{v}(v-s)^{\alpha_{1}-1}\left(\mathfrak{f}_{0}^{i}-\epsilon\right) \varphi_{\varrho_{1}}(x(s)+y(s)) d s\right) d v
\end{aligned}
$$




$$
\begin{aligned}
& +\mu^{\rho_{2}-1} \theta_{1}^{\beta_{1}-1} \int_{\theta_{1}}^{\theta_{2}} \mathcal{J}_{2}(v) \varphi_{\rho_{2}}\left(\frac{1}{\Gamma\left(\alpha_{2}\right)} \int_{\theta_{1}}^{v}(v-s)^{\alpha_{2}-1}\left(\mathfrak{g}_{0}^{i}-\epsilon\right) \varphi_{\varrho_{2}}(x(s)+y(s)) d s\right) d v \\
\geq & \lambda^{\rho_{1}-1} \theta_{1}^{\beta_{1}-1} \int_{\theta_{1}}^{\theta_{2}} \mathcal{J}_{1}(v) \varphi_{\rho_{1}}\left(\frac{1}{\Gamma\left(\alpha_{1}\right)} \int_{\theta_{1}}^{v}(v-s)^{\alpha_{1}-1}\left(\mathfrak{f}_{0}^{i}-\epsilon\right) \varphi_{\varrho_{1}}\left(\zeta\|(x, y)\|_{\mathcal{Y}}\right) d s\right) d v \\
& +\mu^{\rho_{2}-1} \theta_{1}^{\beta_{1}-1} \int_{\theta_{1}}^{\theta_{2}} \mathcal{J}_{2}(v) \varphi_{\rho_{2}}\left(\frac{1}{\Gamma\left(\alpha_{2}\right)} \int_{\theta_{1}}^{v}(v-s)^{\alpha_{2}-1}\left(\mathfrak{g}_{0}^{i}-\epsilon\right) \varphi_{\varrho_{2}}\left(\zeta\|(x, y)\|_{\mathcal{Y}}\right) d s\right) d v \\
= & \zeta \zeta_{1} \varphi_{\rho_{1}}\left(\lambda\left(\mathfrak{f}_{0}^{i}-\epsilon\right)\right)\|(x, y)\|_{\mathcal{Y}} \int_{\theta_{1}}^{\theta_{2}} \mathcal{J}_{1}(v) \frac{1}{\left(\Gamma\left(\alpha_{1}+1\right)\right)^{\rho_{1}-1}}\left(v-\theta_{1}\right)^{\alpha_{1}\left(\rho_{1}-1\right)} d v \\
& +\zeta \zeta_{1} \varphi_{\rho_{2}}\left(\mu\left(\mathfrak{g}_{0}^{i}-\epsilon\right)\right)\|(x, y)\|_{\mathcal{Y}} \int_{\theta_{1}}^{\theta_{2}} J_{2}(v) \frac{1}{\left(\Gamma\left(\alpha_{2}+1\right)\right)^{\rho_{2}-1}}\left(v-\theta_{1}\right)^{\alpha_{2}\left(\rho_{2}-1\right)} d v \\
= & {\left[\zeta \zeta_{1} \varphi_{\rho_{1}}\left(\lambda\left(\mathfrak{f}_{0}^{i}-\epsilon\right)\right) \widetilde{L}_{1}+\zeta \zeta_{1} \varphi_{\rho_{2}}\left(\mu\left(\mathfrak{g}_{0}^{i}-\epsilon\right)\right) \widetilde{L}_{2}\right]\|(x, y)\|_{\mathcal{Y}} } \\
\geq & {\left[a c_{1}+a\left(1-c_{1}\right)\right]\|(x, y)\|_{\mathcal{Y}}=a\|(x, y)\|_{\mathcal{Y}} . }
\end{aligned}
$$

Then $\left\|\mathcal{A}_{1}(x, y)\right\| \geq \mathcal{A}_{1}(x, y)\left(\theta_{1}\right) \geq a\|(x, y)\|_{\mathcal{Y}}$.

In a similar manner we find

$$
\begin{aligned}
& \mathcal{A}_{2}(x, y)\left(\theta_{1}\right) \\
& \geq \zeta \zeta_{2} \varphi_{\rho_{1}}\left(\lambda\left(\mathfrak{f}_{0}^{i}-\epsilon\right)\right)\|(x, y)\|_{\mathcal{Y}} \int_{\theta_{1}}^{\theta_{2}} \mathcal{J}_{3}(v) \frac{1}{\left(\Gamma\left(\alpha_{1}+1\right)\right)^{\rho_{1}-1}}\left(v-\theta_{1}\right)^{\alpha_{1}\left(\rho_{1}-1\right)} d v \\
& \quad+\zeta \zeta_{2} \varphi_{\rho_{2}}\left(\mu\left(\mathfrak{g}_{0}^{i}-\epsilon\right)\right)\|(x, y)\|_{\mathcal{Y}} \int_{\theta_{1}}^{\theta_{2}} J_{4}(v) \frac{1}{\left(\Gamma\left(\alpha_{2}+1\right)\right)^{\rho_{2}-1}}\left(v-\theta_{1}\right)^{\alpha_{2}\left(\rho_{2}-1\right)} d v \\
& =\left[\zeta \zeta_{2} \varphi_{\rho_{1}}\left(\lambda\left(\mathfrak{f}_{0}^{i}-\epsilon\right)\right) \widetilde{L}_{3}+\zeta \zeta_{2} \varphi_{\rho_{2}}\left(\mu\left(\mathfrak{g}_{0}^{i}-\epsilon\right)\right) \widetilde{L}_{4}\right]\|(x, y)\|_{\mathcal{Y}} \\
& \geq\left[(1-a) c_{2}+(1-a)\left(1-c_{2}\right)\right]\|(x, y)\|_{\mathcal{Y}}=(1-a)\|(x, y)\|_{\mathcal{Y}} .
\end{aligned}
$$

So, $\left\|\mathcal{A}_{2}(x, y)\right\| \geq \mathcal{A}_{2}(x, y)\left(\theta_{1}\right) \geq(1-a)\|(x, y)\|_{\mathcal{Y}}$.

Then, for $(x, y) \in \mathcal{P} \cap \partial \Lambda_{3}$, we conclude

$$
\|\mathcal{A}(x, y)\|_{\mathcal{Y}}=\left\|\mathcal{A}_{1}(x, y)\right\|+\left\|\mathcal{A}_{2}(x, y)\right\| \geq a\|(x, y)\|_{\mathcal{Y}}+(1-a)\|(x, y)\|_{\mathcal{Y}}=\|(x, y)\|_{\mathcal{Y}}
$$

We introduce now the functions $\mathfrak{f}^{*}, \mathfrak{g}^{*}:[0,1] \times \mathbb{R}_{+} \rightarrow \mathbb{R}_{+}$by $\mathfrak{f}^{*}(t, u)=\max _{0 \leq x+y \leq u} f(t, x, y)$, $g^{*}(t, u)=\max _{0 \leq x+y \leq u} g(t, x, y)$ for all $t \in[0,1]$ and $u \in \mathbb{R}_{+}$. Then

$$
f(t, x, y) \leq \mathfrak{f}^{*}(t, u), \quad g(t, x, y) \leq \mathfrak{g}^{*}(t, u), \quad \forall t \in[0,1], x, y \geq 0, x+y \leq u .
$$

The functions $\mathfrak{f}^{*}(t, \cdot), \mathfrak{g}^{*}(t, \cdot)$ are nondecreasing for every $t \in[0,1]$ and they satisfy the conditions

$$
\limsup _{u \rightarrow \infty} \max _{t \in[0,1]} \frac{\mathfrak{f}^{*}(t, u)}{\varphi_{\varrho_{1}}(u)}=\mathfrak{f}_{\infty}^{s}, \quad \limsup _{u \rightarrow \infty} \max _{t \in[0,1]} \frac{\mathfrak{g}^{*}(t, u)}{\varphi_{\varrho_{2}}(u)}=\mathfrak{g}_{\infty}^{s} .
$$

Then for $\epsilon>0$ there exists $r_{4}^{\prime}>0$ such that for all $u \geq r_{4}^{\prime}$ and $t \in[0,1]$ we obtain

$$
\frac{\mathfrak{f}^{*}(t, u)}{\varphi_{\varrho_{1}}(u)} \leq \limsup _{u \rightarrow \infty} \max _{t \in[0,1]} \frac{f^{*}(t, u)}{\varphi_{\varrho_{1}}(u)}+\epsilon=\mathfrak{f}_{\infty}^{s}+\epsilon
$$




$$
\frac{\mathfrak{g}^{*}(t, u)}{\varphi_{\varrho_{2}}(u)} \leq \limsup _{u \rightarrow \infty} \max _{t \in[0,1]} \frac{\mathfrak{g}^{*}(t, u)}{\varphi_{\varrho_{2}}(u)}+\epsilon=\mathfrak{g}_{\infty}^{s}+\epsilon
$$

and hence $\mathfrak{f}^{*}(t, u) \leq\left(\mathfrak{f}_{\infty}^{s}+\epsilon\right) \varphi_{\varrho_{1}}(u)$ and $\mathfrak{g}^{*}(t, u) \leq\left(\mathfrak{g}_{\infty}^{s}+\epsilon\right) \varphi_{\varrho_{2}}(u)$.

We consider $r_{4}=\max \left\{2 r_{3}, r_{4}^{\prime}\right\}$ and we introduce the set $\Lambda_{4}=\left\{(x, y) \in \mathcal{Y},\|(x, y)\|_{\mathcal{Y}}<r_{4}\right\}$. Let $(x, y) \in \mathcal{P} \cap \partial \Lambda_{4}$. By the definitions of $\mathfrak{f}^{*}$ and $\mathfrak{g}^{*}$ we deduce

$$
\begin{aligned}
& f(t, x(t), y(t)) \leq \mathfrak{f}^{*}\left(t,\|(x, y)\|_{\mathcal{Y}}\right), \\
& g(t, x(t), y(t)) \leq \mathfrak{g}^{*}\left(t,\|(x, y)\|_{\mathcal{Y}}\right), \quad \forall t \in[0,1] .
\end{aligned}
$$

Then for all $t \in[0,1]$ we find

$$
\begin{aligned}
\mathcal{A}_{1}(x, y)(t) & \\
\leq & \lambda^{\rho_{1}-1} \int_{0}^{1} \mathcal{J}_{1}(v) \varphi_{\rho_{1}}\left(\frac{1}{\Gamma\left(\alpha_{1}\right)} \int_{0}^{v}(v-s)^{\alpha_{1}-1} f(s, x(s), y(s)) d s\right) d v \\
& +\mu^{\rho_{2}-1} \int_{0}^{1} \mathcal{J}_{2}(v) \varphi_{\rho_{2}}\left(\frac{1}{\Gamma\left(\alpha_{2}\right)} \int_{0}^{v}(v-s)^{\alpha_{2}-1} g(s, x(s), y(s)) d s\right) d v \\
\leq & \lambda^{\rho_{1}-1} \int_{0}^{1} \mathcal{J}_{1}(v) \varphi_{\rho_{1}}\left(\frac{1}{\Gamma\left(\alpha_{1}\right)} \int_{0}^{v}(v-s)^{\alpha_{1}-1} \mathfrak{f}^{*}\left(s,\|(x, y)\|_{\mathcal{Y}}\right) d s\right) d v \\
& +\mu^{\rho_{2}-1} \int_{0}^{1} \mathcal{J}_{2}(v) \varphi_{\rho_{2}}\left(\frac{1}{\Gamma\left(\alpha_{2}\right)} \int_{0}^{v}(v-s)^{\alpha_{2}-1} \mathfrak{g}^{*}\left(s,\|(x, y)\|_{\mathcal{Y}}\right) d s\right) d v \\
\leq & \lambda^{\rho_{1}-1} \int_{0}^{1} \mathcal{J}_{1}(v) \varphi_{\rho_{1}}\left(\frac{1}{\Gamma\left(\alpha_{1}\right)} \int_{0}^{v}(v-s)^{\alpha_{1}-1}\left(\mathfrak{f}_{\infty}^{s}+\epsilon\right) \varphi_{\varrho_{1}}\left(\|(x, y)\|_{\mathcal{Y}}\right) d s\right) d v \\
& +\mu^{\rho_{2}-1} \int_{0}^{1} \mathcal{J}_{2}(v) \varphi_{\rho_{2}}\left(\frac{1}{\Gamma\left(\alpha_{2}\right)} \int_{0}^{v}(v-s)^{\alpha_{2}-1}\left(\mathfrak{g}_{\infty}^{s}+\epsilon\right) \varphi_{\varrho_{2}}\left(\|(x, y)\|_{\mathcal{Y}}\right) d s\right) d v \\
= & \varphi_{\rho_{1}}\left(\lambda\left(\mathfrak{f}_{\infty}^{s}+\epsilon\right)\right)\|(x, y)\|_{\mathcal{Y}} \int_{0}^{1} \frac{1}{\left(\Gamma\left(\alpha_{1}+1\right)\right)^{\rho_{1}-1}} v^{\alpha_{1}\left(\rho_{1}-1\right)} \mathcal{J}_{1}(v) d v \\
& +\varphi_{\rho_{2}}\left(\mu\left(\mathfrak{g}_{\infty}^{s}+\epsilon\right)\right)\|(x, y)\|_{\mathcal{Y}} \int_{0}^{1} \frac{1}{\left(\Gamma\left(\alpha_{2}+1\right)\right)^{\rho_{2}-1}} v^{\alpha_{2}\left(\rho_{2}-1\right)} \mathcal{J}_{2}(v) d v \\
= & {\left[\varphi_{\rho_{1}}\left(\lambda\left(\mathfrak{f}_{\infty}^{s}+\epsilon\right)\right) L_{1}+\varphi_{\rho_{2}}\left(\mu\left(\mathfrak{g}_{\infty}^{s}+\epsilon\right)\right) L_{2}\right]\|(x, y)\|_{\mathcal{Y}} } \\
\leq & {\left[b c_{3}+b\left(1-c_{3}\right)\right]\|(x, y)\|_{\mathcal{Y}}=b\|(x, y)\|_{\mathcal{Y}} }
\end{aligned}
$$

and so $\left\|\mathcal{A}_{1}(x, y)\right\| \leq b\|(x, y)\|_{\mathcal{Y}}$.

In a similar manner, we obtain

$$
\begin{aligned}
\mathcal{A}_{2}(x, y)(t) \leq & \varphi_{\rho_{1}}\left(\lambda\left(\mathfrak{f}_{\infty}^{s}+\epsilon\right)\right)\|(x, y)\|_{\mathcal{Y}} \int_{0}^{1} \frac{1}{\left(\Gamma\left(\alpha_{1}+1\right)\right)^{\rho_{1}-1}} v^{\alpha_{1}\left(\rho_{1}-1\right)} \mathcal{J}_{3}(v) d v \\
& +\varphi_{\rho_{2}}\left(\mu\left(\mathfrak{g}_{\infty}^{s}+\epsilon\right)\right)\|(x, y)\|_{\mathcal{Y}} \int_{0}^{1} \frac{1}{\left(\Gamma\left(\alpha_{2}+1\right)\right)^{\rho_{2}-1}} v^{\alpha_{2}\left(\rho_{2}-1\right)} \mathcal{J}_{4}(v) d v \\
= & {\left[\varphi_{\rho_{1}}\left(\lambda\left(\mathfrak{f}_{\infty}^{s}+\epsilon\right)\right) L_{3}+\varphi_{\rho_{2}}\left(\mu\left(\mathfrak{g}_{\infty}^{s}+\epsilon\right)\right) L_{4}\right]\|(x, y)\|_{\mathcal{Y}} } \\
\leq & {\left[(1-b) c_{4}+(1-b)\left(1-c_{4}\right)\right]\|(x, y)\|_{\mathcal{Y}}=(1-b)\|(x, y)\|_{\mathcal{Y}} }
\end{aligned}
$$

and then $\left\|\mathcal{A}_{2}(x, y)\right\| \leq(1-b)\|(x, y)\| \mathcal{Y}$. 
Therefore, for $(x, y) \in \mathcal{P} \cap \partial \Lambda_{4}$, we deduce

$$
\|\mathcal{A}(x, y)\|_{\mathcal{Y}}=\left\|\mathcal{A}_{1}(x, y)\right\|+\left\|\mathcal{A}_{2}(x, y)\right\| \leq b\|(x, y)\|_{\mathcal{Y}}+(1-b)\|(x, y)\|_{\mathcal{Y}}=\|(x, y)\|_{\mathcal{Y}} .
$$

By using Lemma 3.1, Eqs. (23), (24) and the Guo-Krasnosel'skii fixed point theorem, we conclude that $\mathcal{A}$ has a fixed point $(x, y) \in \mathcal{P} \cap\left(\bar{\Lambda}_{4} \backslash \Lambda_{3}\right)$, which is a positive solution of problem (1), (2).

Case (6). We have $\mathfrak{f}_{\infty}^{s} \in(0, \infty), \mathfrak{g}_{\infty}^{s}=0$ and $\mathfrak{g}_{0}^{i}=\infty$. Let $\lambda \in(0, \widetilde{E})$ and $\mu \in(0, \infty)$. We choose $\widetilde{c}_{3} \in\left(\varphi_{\rho_{1}}\left(\lambda f_{\infty}^{s}\right) \frac{L_{1}}{b}, 1\right)$ and $\widetilde{c}_{4} \in\left(\varphi_{\rho_{1}}\left(\lambda f_{\infty}^{s}\right) \frac{L_{3}}{1-b}, 1\right)$. The choice of $\widetilde{c}_{3}$ and $\widetilde{c}_{4}$ is possible because $\lambda<\frac{1}{\mathrm{f}_{\infty}^{s}} \varphi_{\varrho_{1}}\left(\frac{b}{L_{1}}\right)$ and $\lambda<\frac{1}{\mathrm{f}_{\infty}^{s}} \varphi_{\varrho_{1}}\left(\frac{1-b}{L_{3}}\right)$. Let $\epsilon>0$ be such that

$$
\begin{aligned}
& \lambda \leq \min \left\{\frac{1}{\mathfrak{f}_{\infty}^{s}+\epsilon} \varphi_{\varrho_{1}}\left(\frac{b \widetilde{c}_{3}}{L_{1}}\right), \frac{1}{\mathfrak{f}_{\infty}^{s}+\epsilon} \varphi_{\varrho_{1}}\left(\frac{(1-b) \widetilde{c}_{4}}{L_{3}}\right)\right\}, \\
& \varepsilon \varphi_{\varrho_{2}}\left(\frac{1}{\zeta \zeta_{1} \widetilde{L}_{2}}\right) \leq \mu \leq \min \left\{\frac{1}{\epsilon} \varphi_{\varrho_{2}}\left(\frac{b\left(1-\widetilde{c}_{3}\right)}{L_{2}}\right), \frac{1}{\epsilon} \varphi_{\varrho_{2}}\left(\frac{(1-b)\left(1-\widetilde{c}_{4}\right)}{L_{4}}\right)\right\} .
\end{aligned}
$$

By $(I 2)$ and the definition of $\mathfrak{g}_{0}^{i}$ we find that there exists $r_{3}>0$ such that

$$
g(t, x, y) \geq \frac{1}{\epsilon} \varphi_{\varrho_{2}}(x+y), \quad \forall t \in\left[\theta_{1}, \theta_{2}\right], x, y \geq 0, x+y \leq r_{3} .
$$

We define the set $\Lambda_{3}=\left\{(x, y) \in \mathcal{Y},\|(x, y)\| \mathcal{Y}<r_{3}\right\}$. Let $(x, y) \in \mathcal{P} \cap \partial \Lambda_{3}$, that is $\|x\|+\|y\|=$ $r_{3}$. Because $x(t)+y(t) \leq\|x\|+\|y\|=r_{3}$ for all $t \in[0,1]$, then by Lemma 2.5 , we find

$$
\begin{aligned}
& \mathcal{A}_{1}(x, y)\left(\theta_{1}\right) \\
& \geq \lambda^{\rho_{1}-1} \int_{0}^{1} \theta_{1}^{\beta_{1}-1} \mathcal{J}_{1}(v) \varphi_{\rho_{1}}\left(I_{0+}^{\alpha_{1}} f(v, x(v), y(v))\right) d v \\
& \quad+\mu^{\rho_{2}-1} \int_{0}^{1} \theta_{1}^{\beta_{1}-1} \mathcal{J}_{2}(v) \varphi_{\rho_{2}}\left(I_{0+}^{\alpha_{2}} g(v, x(v), y(v))\right) d v \\
& \geq \mu^{\rho_{2}-1} \theta_{1}^{\beta_{1}-1} \int_{\theta_{1}}^{\theta_{2}} \mathcal{J}_{2}(s) \varphi_{\rho_{2}}\left(\frac{1}{\Gamma\left(\alpha_{2}\right)} \int_{\theta_{1}}^{v}(v-s)^{\alpha_{2}-1} g(s, x(s), y(s)) d s\right) d v \\
& \geq \mu^{\rho_{2}-1} \theta_{1}^{\beta_{1}-1} \int_{\theta_{1}}^{\theta_{2}} \mathcal{J}_{2}(v) \varphi_{\rho_{2}}\left(\frac{1}{\Gamma\left(\alpha_{2}\right)} \int_{\theta_{1}}^{v}(v-s)^{\alpha_{2}-1} \frac{1}{\epsilon} \varphi_{\varrho_{2}}(x(s)+y(s)) d s\right) d v \\
& \geq \mu^{\rho_{2}-1} \theta_{1}^{\beta_{1}-1} \int_{\theta_{1}}^{\theta_{2}} \mathcal{J}_{2}(v) \varphi_{\rho_{2}}\left(\frac{1}{\Gamma\left(\alpha_{2}\right)} \int_{\theta_{1}}^{v}(v-s)^{\alpha_{2}-1} \frac{1}{\epsilon} \varphi_{\varrho_{2}}\left(\zeta\|(x, y)\|_{\mathcal{Y}}\right) d s\right) d v \\
& =\zeta \zeta_{1} \varphi_{\rho_{2}}\left(\frac{\mu}{\epsilon}\right)\|(x, y)\|_{\mathcal{Y}} \widetilde{L}_{2} \geq\|(x, y)\|_{\mathcal{Y}} .
\end{aligned}
$$

Hence we deduce $\left\|\mathcal{A}_{1}(x, y)\right\| \geq \mathcal{A}_{1}(x, y)\left(\theta_{1}\right) \geq\|(x, y)\|_{\mathcal{Y}}$ and $\|\mathcal{A}(x, y)\|_{\mathcal{Y}} \geq\left\|\mathcal{A}_{1}(x, y)\right\| \geq$ $\|(x, y)\|_{\mathcal{Y}}$.

For the second part of the proof, we use the functions $\mathfrak{f}^{*}$ and $\mathfrak{g}^{*}$ from Case (1), which satisfy here the conditions

$$
\limsup _{u \rightarrow \infty} \max _{t \in[0,1]} \frac{\mathfrak{f}^{*}(t, u)}{\varphi_{\varrho_{1}}(u)}=\mathfrak{f}_{\infty}^{s}, \quad \lim _{u \rightarrow \infty} \max _{t \in[0,1]} \frac{\mathfrak{g}^{*}(t, u)}{\varphi_{\varrho_{2}}(u)}=0 .
$$


Then for $\epsilon>0$ there exists $r_{4}^{\prime}>0$ such that for all $u \geq r_{4}^{\prime}$ and $t \in[0,1]$ we obtain

$$
\begin{aligned}
& \frac{\mathfrak{f}^{*}(t, u)}{\varphi_{\varrho_{1}}(u)} \leq \limsup _{u \rightarrow \infty} \max _{t \in[0,1]} \frac{\mathfrak{f}^{*}(t, u)}{\varphi_{\varrho_{1}}(u)}+\epsilon=\mathfrak{f}_{\infty}^{s}+\epsilon, \\
& \frac{\mathfrak{g}^{*}(t, u)}{\varphi_{\varrho_{2}}(u)} \leq \lim _{u \rightarrow \infty} \max _{t \in[0,1]} \frac{\mathfrak{g}^{*}(t, u)}{\varphi_{\varrho_{2}}(u)}+\epsilon=\epsilon,
\end{aligned}
$$

and hence $f^{*}(t, u) \leq\left(\mathfrak{f}_{\infty}^{s}+\epsilon\right) \varphi_{\varrho_{1}}(u)$ and $g^{*}(t, u) \leq \epsilon \varphi_{\varrho_{2}}(u)$.

We define $r_{4}=\max \left\{2 r_{3}, r_{4}^{\prime}\right\}$ and we introduce the set $\Lambda_{4}=\left\{(x, y) \in \mathcal{Y},\|(x, y)\|_{\mathcal{Y}}<r_{4}\right\}$. Let $(x, y) \in \mathcal{P} \cap \partial \Lambda_{4}$. By the definitions of $\mathfrak{f}^{*}$ and $\mathfrak{g}^{*}$ we deduce Eq. (23). Besides, in a similar manner to that used in the proof of Case (1), we find

$$
\begin{aligned}
\mathcal{A}_{1}(x, y)(t) & \leq \varphi_{\rho_{1}}\left(\lambda\left(\mathfrak{f}_{\infty}^{s}+\epsilon\right)\right) L_{1}\|(x, y)\|_{\mathcal{Y}}+\varphi_{\rho_{2}}(\mu \epsilon) L_{2}\|(x, y)\|_{\mathcal{Y}} \\
& \leq\left[b \widetilde{c}_{3}+b\left(1-\widetilde{c}_{3}\right)\right]\|(x, y)\|_{\mathcal{Y}}=b\|(x, y)\|_{\mathcal{Y}}, \quad \forall t \in[0,1], \\
\mathcal{A}_{2}(x, y)(t) & \leq \varphi_{\rho_{1}}\left(\lambda\left(\mathfrak{f}_{\infty}^{s}+\epsilon\right)\right) L_{3}\|(x, y)\|_{\mathcal{Y}}+\varphi_{\rho_{2}}(\mu \epsilon) L_{4}\|(x, y)\|_{\mathcal{Y}} \\
& \leq\left[(1-b) \widetilde{c}_{4}+(1-b)\left(1-\widetilde{c}_{4}\right)\right]\|(x, y)\|_{\mathcal{Y}}=(1-b)\|(x, y)\|_{\mathcal{Y}}, \quad \forall t \in[0,1],
\end{aligned}
$$

and then $\|\mathcal{A}(x, y)\|_{\mathcal{Y}} \leq b\|(x, y)\|_{\mathcal{Y}}+(1-b)\|(x, y)\| \mathcal{Y}=\|(x, y)\|_{\mathcal{Y}}$.

Therefore we obtain the conclusion of the theorem.

\section{Nonexistence of the positive solutions}

In this section we give intervals for $\lambda$ and $\mu$ for which there exist no positive solutions of problem (1), (2). By using similar arguments to those used in the proofs of Theorems 4.14.4 from [30], we obtain the following theorems for problem (1), (2).

Theorem 4.1 Assume that (I1) and (I2) hold. If there exist positive numbers $T_{1}, T_{2}$ such that

$$
f(t, x, y) \leq T_{1} \varphi_{\varrho_{1}}(x+y), \quad g(t, x, y) \leq T_{2} \varphi_{\varrho_{2}}(x+y), \quad \forall t \in[0,1], x, y \geq 0,
$$

then there exist positive constants $\lambda_{0}$ and $\mu_{0}$ such that for every $\lambda \in\left(0, \lambda_{0}\right)$ and $\mu \in\left(0, \mu_{0}\right)$ the boundary value problem (1), (2) has no positive solution.

In the proof of Theorem 4.1 we define $\lambda_{0}=\min \left\{\left(T_{1} \varphi_{\varrho_{1}}\left(4 L_{1}\right)\right)^{-1},\left(T_{1} \varphi_{\varrho_{1}}\left(4 L_{3}\right)\right)^{-1}\right\}$ and $\mu_{0}=$ $\min \left\{\left(T_{2} \varphi_{\varrho_{2}}\left(4 L_{2}\right)\right)^{-1},\left(T_{2} \varphi_{\varrho_{2}}\left(4 L_{4}\right)\right)^{-1}\right\}$, where $L_{i}, i=1, \ldots, 4$, are given by $(19)$.

Remark 4.1 If $\mathfrak{f}_{0}^{s}, \mathfrak{g}_{0}^{s}, \mathfrak{f}_{\infty}^{s}, \mathfrak{g}_{\infty}^{s}<\infty$, then there exist positive constants $T_{1}, T_{2}$ such that relation (25) holds, and so we obtain the conclusion of Theorem 4.1.

Theorem 4.2 Assume that (I1) and (I2) hold. If there exist positive numbers $\theta_{1}, \theta_{2}$ with $0<\theta_{1}<\theta_{2} \leq 1$ and $q_{1}>0$ such that

$$
f(t, x, y) \geq q_{1} \varphi_{\varrho_{1}}(x+y), \quad \forall t \in\left[\theta_{1}, \theta_{2}\right], x, y \geq 0,
$$

then there exists a positive constant $\lambda_{0}^{\prime}$ such that for every $\lambda>\lambda_{0}^{\prime}$ and $\mu>0$, the boundary value problem (1), (2) has no positive solution. 
In the proof of Theorem 4.2 we define $\lambda_{0}^{\prime}=\min \left\{\left(q_{1} \varphi_{\varrho_{1}}\left(\zeta \zeta_{1} \widetilde{L}_{1}\right)\right)^{-1},\left(q_{1} \varphi_{\varrho_{1}}\left(\zeta \zeta_{2} \widetilde{L}_{3}\right)\right)^{-1}\right\}$, where $\widetilde{L}_{1}$ and $\widetilde{L}_{3}$ are given by (19).

Remark 4.2 If for $\theta_{1}, \theta_{2}$ with $0<\theta_{1}<\theta_{2} \leq 1$, we have $\mathfrak{f}_{0}^{i}, \mathfrak{f}_{\infty}^{i}>0$, and $f(t, x, y)>0$ for all $t \in\left[\theta_{1}, \theta_{2}\right]$ and $x, y \geq 0$ with $x+y>0$, then Eq. (26) holds, and therefore we obtain the conclusion of Theorem 4.2.

Theorem 4.3 Assume that (I1) and (I2) hold. If there exist positive numbers $\theta_{1}, \theta_{2}$ with $0<\theta_{1}<\theta_{2} \leq 1$ and $q_{2}>0$ such that

$$
g(t, x, y) \geq q_{2} \varphi_{\varrho_{2}}(x+y), \quad \forall t \in\left[\theta_{1}, \theta_{2}\right], x, y \geq 0,
$$

then there exists a positive constant $\mu_{0}^{\prime}$ such that for every $\mu>\mu_{0}^{\prime}$ and $\lambda>0$ the boundary value problem (1), (2) has no positive solution.

In the proof of Theorem 4.3 we define $\mu_{0}^{\prime}=\min \left\{\left(q_{2} \varphi_{\varrho_{2}}\left(\zeta \zeta_{1} \widetilde{L}_{2}\right)\right)^{-1},\left(q_{2} \varphi_{\varrho_{2}}\left(\zeta \zeta_{2} \widetilde{L}_{4}\right)\right)^{-1}\right\}$, where $\widetilde{L}_{2}$ and $\widetilde{L}_{4}$ are given by (19).

Remark 4.3 If for $\theta_{1}, \theta_{2}$ with $0<\theta_{1}<\theta_{2} \leq 1$, we have $\mathfrak{g}_{0}^{i}, \mathfrak{g}_{\infty}^{i}>0$, and $g(t, x, y)>0$ for all $t \in$ $\left[\theta_{1}, \theta_{2}\right]$ and $x, y \geq 0$ with $x+y>0$, then Eq. (27) holds, and hence we deduce the conclusion of Theorem 4.3.

Theorem 4.4 Assume that (I1) and (I2) hold. If there exist positive numbers $\theta_{1}, \theta_{2}$ with $0<\theta_{1}<\theta_{2} \leq 1$ and $q_{1}, q_{2}>0$ such that

$$
f(t, x, y) \geq q_{1} \varphi_{\varrho_{1}}(x+y), \quad g(t, x, y) \geq q_{2} \varphi_{\varrho_{2}}(x+y), \quad \forall t \in\left[\theta_{1}, \theta_{2}\right], x, y \geq 0,
$$

then there exist positive constants $\tilde{\lambda}_{0}$ and $\tilde{\mu}_{0}$ such that for every $\lambda>\widetilde{\lambda}_{0}$ and $\mu>\widetilde{\mu}_{0}$ the boundary value problem (1), (2) has no positive solution.

In the proof of Theorem 4.4 we define $\widetilde{\lambda}_{0}=\left(q_{1} \varphi_{\varrho_{1}}\left(2 \zeta \zeta_{1} \widetilde{L}_{1}\right)\right)^{-1}$ and $\tilde{\mu}_{0}=\left(q_{2} \varphi_{\varrho_{2}}\left(2 \zeta \zeta_{2} \widetilde{L}_{4}\right)\right)^{-1}$.

Remark 4.4 If for $\theta_{1}, \theta_{2}$ with $0<\theta_{1}<\theta_{2} \leq 1$, we have $\mathfrak{f}_{0}^{i}, \mathfrak{f}_{\infty}^{i}, \mathfrak{g}_{0}^{i}, \mathfrak{g}_{\infty}^{i}>0$, and $f(t, x, y)>0$ and $g(t, x, y)>0$ for all $t \in\left[\theta_{1}, \theta_{2}\right]$ and $x, y \geq 0$ with $x+y>0$, then Eq. (28) holds, and so we obtain the conclusion of Theorem 4.4.

\section{An example}

Let $\alpha_{1}=1 / 2, \alpha_{2}=1 / 3, \beta_{1}=10 / 3, n=4, \beta_{2}=17 / 4, m=5, p=2, q=1, \delta_{0}=11 / 5, \gamma_{1}=5 / 6$, $\gamma_{2}=3 / 2, \gamma_{0}=9 / 8, \delta_{1}=3 / 7, H_{1}(t)=4 t$ for all $t \in[0,1], H_{2}(t)=\{1, t \in[0,1 / 4) ; 8, t \in[1 / 4,1]\}$, $K_{1}(t)=\{1, t \in[0,1 / 2) ; 3, t \in[1 / 2,1]\}, \varrho_{1}=5, \rho_{1}=5 / 4, \varrho_{2}=3, \rho_{2}=3 / 2, \varphi_{\varrho_{1}}(s)=|s|^{3} s, \varphi_{\rho_{1}}(s)=$ $|s|^{-3 / 4} s, \varphi_{\varrho_{2}}(s)=|s| s, \varphi_{\rho_{2}}(s)=|s|^{-1 / 2} s$.

We consider the system of fractional differential equations

$$
\left\{\begin{array}{l}
D_{0+}^{1 / 2}\left(\varphi_{5}\left(D_{0+}^{10 / 3} x(t)\right)\right)+\lambda(3-t)^{a_{0}}\left(e^{(x(t)+y(t))^{4}}-1\right)=0, \quad t \in(0,1), \\
D_{0+}^{1 / 3}\left(\varphi_{3}\left(D_{0+}^{17 / 4} y(t)\right)\right)+\mu(t+2)^{b_{0}}\left(x^{3}(t)+y^{3}(t)\right)=0, \quad t \in(0,1),
\end{array}\right.
$$


with the coupled nonlocal boundary conditions

$$
\left\{\begin{array}{l}
x(0)=x^{\prime}(0)=x^{\prime \prime}(0)=0, \quad D_{0+}^{10 / 3} x(0)=0, \\
D_{0+}^{9 / 8} x(1)=4 \int_{0}^{1} D_{0+}^{5 / 6} y(t) d t+7 D_{0+}^{3 / 2} y\left(\frac{1}{4}\right), \\
y(0)=y^{\prime}(0)=y^{\prime \prime}(0)=y^{\prime \prime \prime}(0)=0, \quad D_{0+}^{17 / 4} y(0)=0, \\
D_{0+}^{11 / 5} y(1)=2 D_{0+}^{3 / 7} x\left(\frac{1}{2}\right),
\end{array}\right.
$$

where $a_{0}, b_{0}>0$.

Here we have $f(t, x, y)=(3-t)^{a_{0}}\left(e^{(x+y)^{4}}-1\right), g(t, x, y)=(t+2)^{b_{0}}\left(x^{3}+y^{3}\right)$ for all $t \in[0,1]$ and $x, y \geq 0$. We obtain $\Delta \approx 15.18283383>0$, and then the assumptions (I1) and (I2) are satisfied. Besides, we find

$$
\begin{aligned}
& g_{1}(t, s)=\frac{1}{\Gamma(10 / 3)} \begin{cases}t^{7 / 3}(1-s)^{29 / 24}-(t-s)^{7 / 3}, & 0 \leq s \leq t \leq 1, \\
t^{7 / 3}(1-s)^{29 / 24}, & 0 \leq t \leq s \leq 1,\end{cases} \\
& g_{11}(t, s)=\frac{1}{\Gamma(61 / 21)} \begin{cases}t^{40 / 21}(1-s)^{29 / 24}-(t-s)^{40 / 21}, & 0 \leq s \leq t \leq 1, \\
t^{40 / 21}(1-s)^{29 / 24}, & 0 \leq t \leq s \leq 1,\end{cases} \\
& g_{2}(t, s)=\frac{1}{\Gamma(17 / 4)} \begin{cases}t^{13 / 4}(1-s)^{21 / 20}-(t-s)^{13 / 4}, & 0 \leq s \leq t \leq 1, \\
t^{13 / 4}(1-s)^{21 / 20}, & 0 \leq t \leq s \leq 1,\end{cases} \\
& g_{21}(t, s)=\frac{1}{\Gamma(41 / 12)} \begin{cases}t^{29 / 12}(1-s)^{21 / 20}-(t-s)^{29 / 12}, & 0 \leq s \leq t \leq 1, \\
t^{29 / 12}(1-s)^{21 / 20}, & 0 \leq t \leq s \leq 1,\end{cases} \\
& g_{22}(t, s)=\frac{1}{\Gamma(11 / 4)} \begin{cases}t^{7 / 4}(1-s)^{21 / 20}-(t-s)^{7 / 4}, & 0 \leq s \leq t \leq 1, \\
t^{7 / 4}(1-s)^{21 / 20}, & 0 \leq t \leq s \leq 1,\end{cases} \\
& \mathcal{G}_{1}(t, s)=g_{1}(t, s)+\frac{2 t^{7 / 3}}{\Delta}\left[\frac{48 \Gamma(17 / 4)}{41 \Gamma(41 / 12)}+7\left(\frac{1}{4}\right)^{7 / 4} \frac{\Gamma(17 / 4)}{\Gamma(11 / 4)}\right] g_{11}\left(\frac{1}{2}, s\right), \\
& \mathcal{G}_{2}(t, s)=\frac{t^{7 / 3} \Gamma(17 / 4)}{\Delta \Gamma(41 / 20)}\left[4 \int_{0}^{1} g_{21}(\tau, s) d \tau+7 g_{22}\left(\frac{1}{4}, s\right)\right], \\
& \mathcal{G}_{3}(t, s)=\frac{2 t^{13 / 4} \Gamma(10 / 3)}{\Delta \Gamma(53 / 24)} g_{11}\left(\frac{1}{2}, s\right), \\
& \mathcal{G}_{4}(t, s)=g_{2}(t, s)+\frac{t^{13 / 4} \Gamma(10 / 3)}{\Delta \Gamma(61 / 21) 2^{19 / 21}}\left[4 \int_{0}^{1} g_{21}(\tau, s) d \tau+7 g_{22}\left(\frac{1}{4}, s\right)\right],
\end{aligned}
$$

for all $(t, s) \in[0,1] \times[0,1]$. For the functions $h_{1}, h_{2}$ and $\mathcal{J}_{i}, i=1, \ldots, 4$, we obtain

$$
\begin{aligned}
& h_{1}(s)=\frac{1}{\Gamma(10 / 3)}\left[(1-s)^{29 / 24}-(1-s)^{7 / 3}\right], \\
& h_{2}(s)=\frac{1}{\Gamma(17 / 4)}\left[(1-s)^{21 / 20}-(1-s)^{13 / 4}\right],
\end{aligned}
$$

for all $s \in[0,1]$, 


$$
\begin{aligned}
& \mathcal{J}_{1}(s)= \begin{cases}\frac{1}{\Gamma(10 / 3)}\left[(1-s)^{29 / 24}-(1-s)^{7 / 3}\right]+\frac{1}{\Delta}\left[\frac{48 \Gamma(17 / 4)}{41 \Gamma(41 / 12)}+7\left(\frac{1}{4}\right)^{7 / 4} \frac{\Gamma(17 / 4)}{\Gamma(11 / 4)}\right] & \\
\times \frac{2}{\Gamma(61 / 21)}\left[\left(\frac{1}{2}\right)^{40 / 21}(1-s)^{29 / 24}-\left(\frac{1}{2}-s\right)^{40 / 21}\right], & 0 \leq s<\frac{1}{2}, \\
\frac{1}{\Gamma(10 / 3)}\left[(1-s)^{29 / 24}-(1-s)^{7 / 3}\right]+\frac{1}{\Delta}\left[\frac{48 \Gamma(17 / 4)}{41 \Gamma(41 / 12)}+7\left(\frac{1}{4}\right)^{7 / 4} \frac{\Gamma(17 / 4)}{\Gamma(11 / 4)}\right] & \\
\quad \times \frac{2}{\Gamma(61 / 21)}\left(\frac{1}{2}\right)^{40 / 21}(1-s)^{29 / 24}, & \frac{1}{2} \leq s \leq 1,\end{cases}
\end{aligned}
$$

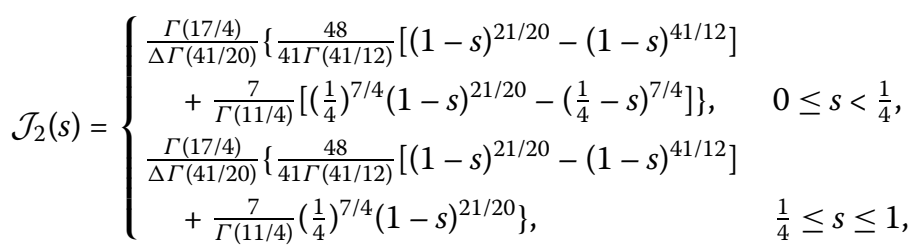

$$
\begin{aligned}
& \mathcal{J}_{3}(s)= \begin{cases}\frac{2 \Gamma(10 / 3)}{\Delta \Gamma(53 / 24) \Gamma(61 / 21)}\left[\left(\frac{1}{2}\right)^{40 / 21}(1-s)^{29 / 24}-\left(\frac{1}{2}-s\right)^{40 / 21}\right], & 0 \leq s<\frac{1}{2}, \\
\frac{2 \Gamma(10 / 3)}{\Delta \Gamma(53 / 24) \Gamma(61 / 21)}\left(\frac{1}{2}\right)^{40 / 21}(1-s)^{29 / 24}, & \frac{1}{2} \leq s \leq 1,\end{cases}
\end{aligned}
$$

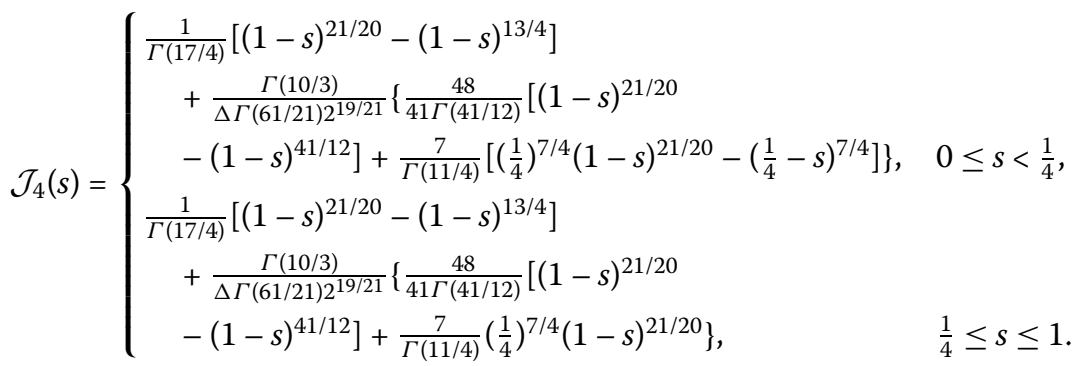

We choose $\theta_{1}=1 / 4$ and $\theta_{2}=3 / 4$, and hence we obtain $\zeta_{1}=(1 / 4)^{7 / 3}, \zeta_{2}=(1 / 4)^{13 / 4}$ and $\zeta=\zeta_{2}$. Besides, we deduce $\mathfrak{f}_{0}^{s}=3^{a_{0}}, \mathfrak{f}_{\infty}^{i}=\infty, \mathfrak{g}_{0}^{s}=0, \mathfrak{g}_{\infty}^{i}=\infty, L_{1} \approx 0.08207184$, $L_{3} \approx 0.01229905, \widetilde{L}_{1} \approx 0.05185073, \widetilde{L}_{3} \approx 0.00775575$.

By Theorem 3.1(7), if we consider $b=1 / 2$, then, for any $\lambda \in(0, E)$ and $\mu \in(0, \infty)$ with $E=3^{-a_{0}}\left(2 L_{1}\right)^{-4}$, the problem (29), (30) has a positive solution $(x(t), y(t)), t \in[0,1]$. For example, if $a_{0}=1$ we find $E \approx 459.179$.

We can also use Theorem 4.2, because $f(t, x, y) \geq q_{1}(x+y)^{4}$ for all $t \in[1 / 4,3 / 4]$ and $x, y \geq 0$, with $q_{1}=(9 / 4)^{a_{0}}$. If $a_{0}=1$, we obtain $\lambda_{0}^{\prime}=\left(q_{1}\left(\zeta \zeta_{1} \widetilde{L}_{1}\right)^{4}\right)^{-1} \approx 1.71714 \times 10^{18}$, and then we deduce then, for every $\lambda>\lambda_{0}^{\prime}$ and $\mu>0$, that the boundary value problem (29), (30) has no positive solution.

Acknowledgements

Not applicable.

Funding

Not applicable.

Abbreviations

Not applicable.

Availability of data and materials

Not applicable.

Ethics approval and consent to participate

Not applicable.

Competing interests

The authors declare that they have no competing interests.

Consent for publication

Not applicable. 
Authors' contributions

All authors read and approved the final manuscript.

\section{Authors' information}

Alexandru Tudorache, Faculty of Automatic Control and Computer Engineering, Gh. Asachi Technical University, Str. Prof.dr.doc. Dimitrie Mangeron, nr.27, lasi 700050, Romania. Rodica Luca, Department of Mathematics, Gh. Asachi Technical University, Blvd. Carol I, nr.11, lasi 700506, Romania.

\section{Author details}

${ }^{1}$ Faculty of Automatic Control and Computer Engineering, Gh. Asachi Technical University, lasi, Romania. ${ }^{2}$ Department of Mathematics, Gh. Asachi Technical University, lasi, Romania.

\section{Publisher's Note}

Springer Nature remains neutral with regard to jurisdictional claims in published maps and institutional affiliations.

Received: 23 April 2020 Accepted: 2 June 2020 Published online: 15 June 2020

\section{References}

1. Agarwal, R.P., Luca, R.: Positive solutions for a semipositone singular Riemann-Liouville fractional differential problem. Int. J. Nonlinear Sci. Numer. Simul. 20(7-8), 823-832 (2019)

2. Ahmad, B., Alsaedi, A., Aljoudi, S., Ntouyas, S.K.: A six-point nonlocal boundary value problem of nonlinear coupled sequential fractional integro-differential equations and coupled integral boundary conditions. J. Appl. Math. Comput. 56(1-2), 367-389 (2018)

3. Ahmad, B., Alsaedi, A., Ntouyas, S.K., Tariboon, J.: Hadamard-Type Fractional Differential Equations, Inclusions and Inequalities. Springer, Cham (2017)

4. Ahmad, B., Luca, R.: Existence of solutions for a sequential fractional integro-differential system with coupled integral boundary conditions. Chaos Solitons Fractals 104, 378-388 (2017)

5. Ahmad, B., Luca, R.: Existence of solutions for a system of fractional differential equations with coupled nonlocal boundary conditions. Fract. Calc. Appl. Anal. 21(2), 423-441 (2018)

6. Ahmad, B., Luca, R.: Existence of solutions for sequential fractional integro-differential equations and inclusions with nonlocal boundary conditions. Appl. Math. Comput. 339, 516-534 (2018)

7. Ahmad, B., Ntouyas, S., Alsaedi, A.: On a coupled system of fractional differential equations with coupled nonlocal and integral boundary conditions. Chaos Solitons Fractals 83, 234-241 (2016)

8. Ahmad, B., Ntouyas, S.K., Alsaedi, A.: Sequential fractional differential equations and inclusions with semi-periodic and nonlocal integro-multipoint boundary conditions. J. King Saud Univ., Sci. 31(2), 184-193 (2019)

9. Arafa, A.A.M., Rida, S.Z., Khalil, M.: Fractional modeling dynamics of HIV and CD4 ${ }^{+}$T-cells during primary infection. Nonlinear Biomed. Phys. 6(1), 1-7 (2012)

10. Baleanu, D., Diethelm, K., Scalas, E., Trujillo, J.J.: Fractional Calculus Models and Numerical Methods. Series on Complexity, Nonlinearity and Chaos. World Scientific, Boston (2012)

11. Das, S.: Functional Fractional Calculus for System Identification and Controls. Springer, New York (2008)

12. Ding, Y., Ye, H.: A fractional-order differential equation model of HIV infection of CD4 ${ }^{+}$T-cells. Math. Comput. Model. 50, 386-392 (2009)

13. Djordjevic, V., Jaric, J., Fabry, B., Fredberg, J., Stamenovic, D.: Fractional derivatives embody essential features of cell rheological behavior. Ann. Biomed. Eng. 31, 692-699 (2003)

14. Ge, Z.M., Ou, C.Y.: Chaos synchronization of fractional order modified Duffing systems with parameters excited by a chaotic signal. Chaos Solitons Fractals 35, 705-717 (2008)

15. Graef, J.R., Kong, L., Kong, Q., Wang, M.: Uniqueness of positive solutions of fractional boundary value problems with non-homogeneous integral boundary conditions. Fract. Calc. Appl. Anal. 15(3), 509-528 (2012)

16. Guo, D., Lakshmikantham, V.: Nonlinear Problems in Abstract Cones. Academic Press, New York (1988)

17. Henderson, J., Luca, R.: Boundary Value Problems for Systems of Differential, Difference and Fractional Equations. Positive Solutions. Elsevier, Amsterdam (2016)

18. Henderson, J., Luca, R.: Positive solutions for a system of semipositone coupled fractional boundary value problems. Bound. Value Probl. 2016, 61 (2016)

19. Henderson, J., Luca, R.: Existence of positive solutions for a singular fractional boundary value problem. Nonlinear Anal., Model. Control 22(1), 99-114 (2017)

20. Henderson, J., Luca, R.: Systems of Riemann-Liouville fractional equations with multi-point boundary conditions. Appl. Math. Comput. 309, 303-323 (2017)

21. Henderson, J., Luca, R., Tudorache, A.: Existence and nonexistence of positive solutions for coupled Riemann-Liouville fractional boundary value problems. Discrete Dyn. Nat. Soc. 2016, Article ID 2823971 (2016)

22. Henderson, J., Luca, R., Tudorache, A.: Existence of positive solutions for a system of fractional boundary value problems. In: Pinelas, S., Dosla, Z., Dosly, O., Kloeden, P.E. (eds.) Differential and Difference Equations with Applications, ICDDEA, Amadora, Portugal, May 2015. Selected Contributions, pp. 349-357. Springer, Berlin (2016)

23. Henderson, J., Luca, R., Tudorache, A.: Existence and nonexistence of positive solutions to a discrete boundary value problem. Carpath. J. Math. 33(2), 181-190 (2017)

24. Jiang, J., Liu, L., Wu, Y.: Symmetric positive solutions to singular system with multi-point coupled boundary conditions. Appl. Math. Comput. 220(1), 536-548 (2013)

25. Jiang, J., Liu, L., Wu, Y.: Positive solutions to singular fractional differential system with coupled boundary conditions. Commun. Nonlinear Sci. Numer. Simul. 18(11), 3061-3074 (2013)

26. Kilbas, A.A., Srivastava, H.M., Trujillo, J.J.: Theory and Applications of Fractional Differential Equations. North-Holland Mathematics Studies, vol. 204. Elsevier, Amsterdam (2006)

27. Klafter, J., Lim, S.C., Metzler, R. (eds.): Fractional Dynamics in Physics World Scientific, Singapore (2011) 
28. Luca, R.: Positive solutions for a system of Riemann-Liouville fractional differential equations with multi-point fractional boundary conditions. Bound. Value Probl. 2017, 102 (2017)

29. Luca, R.: Positive solutions for a system of fractional differential equations with $p$-Laplacian operator and multi-point boundary conditions. Nonlinear Anal., Model. Control 23(5), 771-801 (2018)

30. Luca, R.: On a system of fractional boundary value problems with p-Laplacian operator. Dyn. Syst. Appl. 28(3), 691-713 (2019)

31. Luca, R., Tudorache, A.: Positive solutions to a system of semipositone fractional boundary value problems. Adv. Differ. Equ. 2014, 179 (2014)

32. Metzler, R., Klafter, J.: The random walks guide to anomalous diffusion: a fractional dynamics approach. Phys. Rep. $339,1-77(2000)$

33. Ostoja-Starzewski, M.: Towards thermoelasticity of fractal media. J. Therm. Stresses 30, 889-896 (2007)

34. Podlubny, l.: Fractional Differential Equations. Academic Press, San Diego (1999)

35. Sabatier, J., Agrawal, O.P., Machado, J.A.T. (eds.): Advances in Fractional Calculus: Theoretical Developments and Applications in Physics and Engineering Springer, Dordrecht (2007)

36. Samko, S.G., Kilbas, A.A., Marichev, O.I.: Fractional Integrals and Derivatives. Theory and Applications. Gordon \& Breach, Yverdon (1993)

37. Shen, C., Zhou, H., Yang, L.: Positive solution of a system of integral equations with applications to boundary value problems of differential equations. Adv. Differ. Equ. 2016, 260 (2016)

38. Sokolov, I.M., Klafter, J., Blumen, A.: A fractional kinetics. Phys. Today 55, 48-54 (2002)

39. Wang, Y., Liu, L., Wu, Y.: Positive solutions for a class of higher-order singular semipositone fractional differential systems with coupled integral boundary conditions and parameters. Adv. Differ. Equ. 2014, 268 (2014)

40. Yuan, C.: Two positive solutions for $(n-1,1)$-type semipositone integral boundary value problems for coupled systems of nonlinear fractional differential equations. Commun. Nonlinear Sci. Numer. Simul. 17(2), 930-942 (2012)

41. Yuan, C., Jiang, D., O'Regan, D., Agarwal, R.P.: Multiple positive solutions to systems of nonlinear semipositone fractional differential equations with coupled boundary conditions. Electron. J. Qual. Theory Differ. Equ. 2012, 13 (2012)

\section{Submit your manuscript to a SpringerOpen ${ }^{\circ}$ journal and benefit from:}

- Convenient online submission

- Rigorous peer review

- Open access: articles freely available online

- High visibility within the field

Retaining the copyright to your article

Submit your next manuscript at $\gg$ springeropen.com 